\title{
Optimal Position and Velocity Estimation for Multi-USV Positioning Systems with Range Measurements
}

\author{
Wei Chen, ${ }^{1}$ Ruisheng Sun $\mathbb{D}^{1},{ }^{1}$ and Weisheng Yan $\mathbb{D}^{2}$ \\ ${ }^{1}$ School of Energy and Power Engineering, Nanjing University of Science and Technology, Nanjing, Jiangsu 210094, China \\ ${ }^{2}$ School of Marine Science and Technology, Northwestern Polytechnical University, Xi'an, Shaanxi 710072, China \\ Correspondence should be addressed to Ruisheng Sun; srscom@163.com
}

Received 27 March 2018; Accepted 29 May 2018; Published 10 July 2018

Academic Editor: Liang $\mathrm{Hu}$

Copyright (c) 2018 Wei Chen et al. This is an open access article distributed under the Creative Commons Attribution License, which permits unrestricted use, distribution, and reproduction in any medium, provided the original work is properly cited.

\begin{abstract}
This paper investigates the problem on simultaneously estimating the velocity and position of the target for range-based multi-USV positioning systems. According to the range measurement and kinematics model of the target, we formulate this problem in a mixed linear/nonlinear discrete-time system. In this system, the input and state represent the velocity and position of the target, respectively. We divide the system into two components and propose a three-step minimum variance unbiased simultaneous input and state estimation (SISE) algorithm. First, we estimate the velocity in the local level plane and predict the corresponding position. Then, we estimate the velocity in the heave direction. Finally, we estimate the 3-dimensional (3D) velocity and position. We establish the unbiased conditions of the input and state estimation for the MLBL system. Simulation results illustrate the effectiveness of the problem formulation and demonstrate the performance of the proposed algorithm.
\end{abstract}

\section{Introduction}

Since electromagnetic signal decays quickly in the water, the well-known GPS cannot be used $[1,2]$. The acoustic positioning systems play an important role for underwater positioning [3-5]. These systems are widely applied in many underwater tasks, including salvage operations, minehunting, animal tracking, marine archaeology, oceanographic survey, and military activities. Classical underwater acoustic positioning systems include long baseline (LBL) system, short baseline (SBL) system, and ultrashort baseline (USBL) system [6-8]. Among these systems, LBL system has the best positioning accuracy [9]. However, it has several drawbacks, for example, difficult to obtain the positions of the seabed transponders, fixed and limited positioning regions, and hard to place and recover the transponders [10-12].

Moving long baseline (MLBL) system is a generalization of LBL system by replacing the precalibrated arrays of static transponders with unmanned surface vessels (USVs). Figure 1 shows the schematic of multi-USV positioning system. It overcomes the shortcomings of LBL system described above. The recent researches of MLBL system are concentrated on the positioning algorithms, the optimal formation, and formation control. References [13-15] studied the optimal formation of MLBL system. Accordingly, the optimal range between the USV and the target is studied in [16]. References [17-19] provided some resource-reducing data transmission approaches in the sensor network. References $[20,21]$ studied the formation control of the underwater vehicles. In past few years, many positioning algorithms based on the range measurements have been proposed in the literature, such as least squares (LS) [22], Kalman filtering (KF) $[23,24]$, particle filtering (PF) [25], and maximum likelihood estimation (MLE) [26-28]. In LS and MLE algorithms, the target position is estimated by the current range measurements and unrelated with the velocity of the target. In KF and PF algorithms, the target position is estimated by the current range measurements, the previous position, and the velocity of the target. Among all these positioning algorithms, the $\mathrm{KF}$ and $\mathrm{PF}$ algorithms have better positioning accuracies. In both algorithms, more information, such as the velocity of the target, is used to estimate the position of the target. In some underwater tasks, such as salvage operations and marine archaeology, the velocity of the underwater 


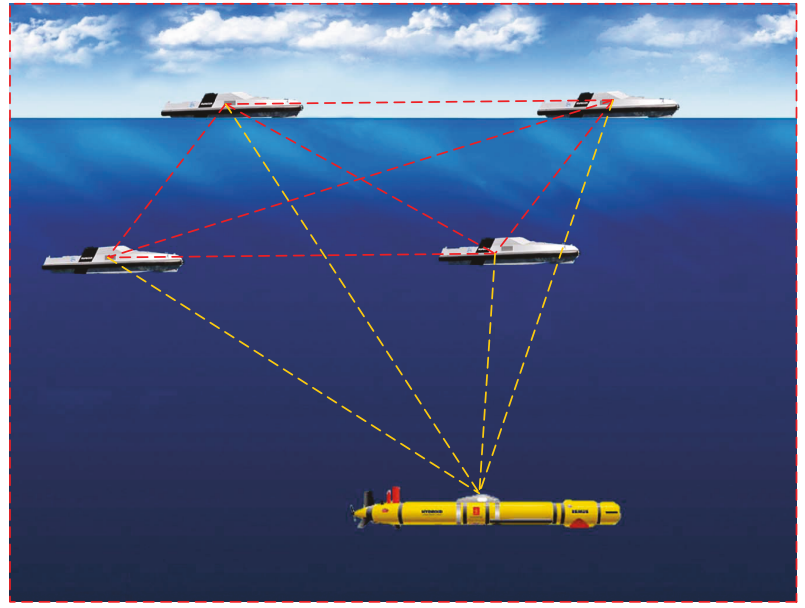

FIGURE 1: MLBL system consists of four USVs [16].

vehicle can be measured by doppler velocity log (DVL) fitted to it, and the velocity of the target is the key to predict the position of the target in advance. However, in other tasks, such as animal tracking, the velocity of the target is hard to be measured. Hence, in this paper, we propose a method to simultaneously estimate the velocity and position of the target based on simultaneous input and state estimation (SISE).

In recent years, the unbiased minimum variance SISE for linear systems has been extensively studied. Li et al. presented extensive reviews for state filtering with unknown inputs [29]. Kitanidis proposed an unbiased recursive filter to estimate the state of linear systems without prior information about the unknown input [30]. Gillijns and De Moor proposed the unbiased minimum variance SISE for linear discrete-time systems with/without direct feedthrough [31, 32]. Floquet and Barbot designed an input and state delayed estimator for discrete-time linear systems even if some wellknown matching condition does not hold [33]. Yong et al. presented an exponentially stable filter for linear discretetime stochastic systems that simultaneously estimates the state and unknown input [34]. Su et al. investigate the properties of the Kalman filter for linear stochastic time-varying systems with partially observed inputs [35]. Fang et al. analysed the stability conditions of SISE algorithms for linear discrete-time systems with/without direct feedthrough [36]. Among all these SISE algorithms, the input is obtained by least square estimation and the state estimation problem is transformed into a KF problem. The main objective of this paper is to design a simultaneous velocity and position estimation method for range-based multi-USV positioning system. The main contributions of this paper are mainly three-fold. First, we formulate the positioning system in a mixed linear/nonlinear discrete-time system. In this system, the velocity and position of the target are seen as the input and state, respectively. Second, a three-step minimum variance unbiased SISE algorithm is proposed by converting the nonlinear measurement equation into two linear measurement equations. Finally, we analyse the estimation conditions for this system.
The remainder of this paper is organized as follows. In Section 2, we formulate the velocity and position estimation for the multi-USV positioning system. The unbiased minimum variance velocity and position estimation algorithms are designed in Sections 3 and 4, respectively. Section 5 derives the unbiased SISE conditions for this system. Section 6 illustrates simulation results to verify the effectiveness of problem formulation and demonstrate the performance of the proposed algorithm. In Section 7, we conclude with a brief discussion of ongoing and future work.

\section{Problem Formulation}

The notations used throughout the paper are as follows. $\mathbb{R}^{n}$ denotes the $n$-dimensional Euclidean space and $\mathbf{I}_{n}$ is the identity matrix of size $n$. For matrix $\mathbf{X}, \mathbf{X}^{\mathrm{T}}$, and $\mathbf{X}^{-1}$ are its transpose and inverse, respectively. We use $\operatorname{rank}(\mathbf{X})$ to denote the rank of $\mathbf{X}$. For random variable $d$, the expectation is denoted by $\mathbb{E}(d)$. We use $\bar{d}$ and $\widehat{d}$ to indicate the prediction and estimation of $d$. Some basic notions from estimation theory are defined as follows.

Definition 1. (see [37]). Let $\widehat{\theta}$ denotes a statistic, one say $\widehat{\theta}$ is an unbiased estimator of $\theta$ if $\mathbb{E}(\theta-\widehat{\theta})=0$.

Definition 2. (see [37]). The estimator $\widehat{\theta}$ is the minimum variance unbiased estimator (MVUE) of $\theta$, if $\widehat{\theta}$ is unbiased, and if the variance of $\widehat{\theta}, \operatorname{var}(\widehat{\theta}):=\mathbb{E}\left((\theta-\widehat{\theta})(\theta-\widehat{\theta})^{\mathrm{T}}\right)$, is less than or equal to the variance of every other unbiased estimator of $\theta$.

Consider an earth fixed reference frame $\{O\}:=\left\{x_{0}, y_{0}\right.$, $\left.z_{0}\right\}$ with $z=0$ on the water surface and the $z$-axis pointing downward from the water surface. Suppose there are $m$ USV to locate the target. The coordinate of the target at stamp $k$ is $\left(x_{k}, y_{k}, z_{k}\right), i=1,2, \ldots, m$. The kinematics models of the target is described as [38]

$$
\begin{aligned}
& x_{k+1}=x_{k}+v_{k}^{x} t_{k}, \\
& y_{k+1}=y_{k}+v_{k}^{y} t_{k}, \\
& z_{k+1}=z_{k}+v_{k}^{z} t_{k},
\end{aligned}
$$

where $t_{k}$ is the sampling period at stamp $k$, and $\left(v_{k}^{x}, v_{k}^{y}, v_{k}^{z}\right)$ is the velocity of the target. Due to the USVs are on the water surface, we have $z_{i, k}=0$. Hence, the coordinate of USV $i$ at stamp $k$ is $\left(x_{i, k}, y_{i, k}, 0\right)$. Define $r_{i, k}$ as the range between the USV $i$ and the target, we have

$$
r_{i, k}^{2}=\left(x_{k}-x_{i, k}\right)^{2}+\left(y_{k}-y_{i, k}\right)^{2}+z_{k}^{2} .
$$

In order to simultaneously estimate the velocity and position of the target, we transform the multi-USV positioning system into a time-variant discrete-time system. The kinematics model of the target and the range measurement are regarded as the process equation and measurement equation, respectively. Combining the kinematics model (1) and the 
range (2), we formulate this system in a mixed linear/nonlinear, time-variant, discrete-time system.

$$
\begin{aligned}
\mathbf{X}_{k+1} & =\mathbf{X}_{k}+T_{k} \mathbf{V}_{k}+w_{k}, \\
r_{k} & =f\left(\mathbf{X}_{k}\right)+\xi_{r, k},
\end{aligned}
$$

with

$$
\begin{aligned}
T_{k} & :=\operatorname{diag}\left[t_{k}, t_{k}, t_{k}\right], \\
f\left(\mathbf{X}_{k}\right) & :=\left[f_{1}, f_{2}, \ldots, f_{m}\right]^{\mathrm{T}}, \\
f_{i} & :=\left(x_{k}-x_{i, k}\right)^{2}+\left(y_{k}-y_{i, k}\right)^{2}+z_{k}^{2},
\end{aligned}
$$

where $\mathbf{X}_{k}:=\left[x_{k}, y_{k}, z_{k}\right]^{\mathrm{T}} \in \mathbb{R}^{3}$ is the state at stamp $k, \mathbf{V}_{k}:=$ $\left[v_{k}^{x}, v_{k}^{y}, v_{k}^{z}\right]^{\mathrm{T}} \in \mathbb{R}^{3}$ is the unknown input. $r_{k}:=\left[r_{1, k}^{2}, \ldots, r_{m, k}^{2}\right]^{\mathrm{T}}$ $\in \mathbb{R}^{m}$ is the measurement. $w_{k} \in \mathbb{R}^{3}$ and $\xi_{r, k}:=\left[\xi_{r_{1}, k}, \ldots\right.$, $\left.\xi_{r_{m}, k}\right]^{\mathrm{T}} \in \mathbb{R}^{m}$ are the noises. According to the error model of range measurement $r_{i, k}[39,40]$, we have

$$
\varepsilon_{i, k}=\left(1+\eta r_{i, k}\right) \varepsilon
$$

where $\varepsilon_{i, k}$ is the measurement error of range measurement $r_{i, k}, \varepsilon$ is a Gaussian stochastic process with $\varepsilon \sim N\left(0, \sigma^{2}\right)$, and $\eta$ is the parameter for the range-dependent error component. Simultaneously, we define

$$
\xi_{r_{i}, k}=\left(1+\eta r_{i, k}\right)^{2} \varepsilon_{r},
$$

where $\varepsilon_{r}$ is a Gaussian stochastic process with $\varepsilon_{r} \sim N\left(0, \sigma_{r}^{2}\right)$.

Since system in (3) and (4) is a mixed linear/nonlinear time-variant discrete-time system, it is hard to simultaneously estimate the state and input. Hence, we design a three-step unbiased minimum variance SISE algorithm to solve this problem.

Define $r_{j, k}$ as the range between the USV $j$ and the target, we have

$$
r_{j, k}^{2}=\left(x_{k}-x_{j, k}\right)^{2}+\left(y_{k}-y_{j, k}\right)^{2}+z_{k}^{2} .
$$

Combining (2) and (8), we have

$$
\gamma_{k}^{i, j}=\alpha_{k}^{i, j} x_{k}+\beta_{k}^{i, j} y_{k}
$$

with

$$
\begin{aligned}
& \gamma_{k}^{i, j}:=r_{i, k}^{2}-r_{j, k}^{2}-x_{i, k}^{2}-y_{i, k}^{2}+x_{j, k}^{2}+y_{j, k}^{2}, \\
& \alpha_{k}^{i, j}:=-2\left(x_{i, k}-x_{j, k}\right), \\
& \beta_{k}^{i, j}:=-2\left(y_{i, k}-y_{j, k}\right) .
\end{aligned}
$$

It follows that

$$
\gamma k=\mathbf{B}_{k} \mathbf{X}_{k}+\xi_{1, k}
$$

where $\gamma k:=\left[\gamma_{k}^{1,2}, \gamma_{k}^{2,3}, \ldots, \gamma_{k}^{m-1, m}\right]^{\mathrm{T}} \in \mathbb{R}^{m-1}$ is the measurement and $\xi_{1, k}:=\left[\xi_{r_{1}, k}-\xi_{r_{2}, k}, \ldots, \xi_{r_{m-1}, k}-\xi_{r_{m}, k}\right]^{\mathrm{T}} \in \mathbb{R}^{m}$ is the zero mean white noise with covariance $\mathbf{R}_{1, k}$. $\mathbf{B}_{k}$ and $\mathbf{R}_{1, k}$ are known matrices with

$$
\begin{aligned}
& \mathbf{B}_{k}:= {\left[\begin{array}{ccc}
\alpha_{k}^{1,2} & \beta_{k}^{1,2} & 0 \\
\alpha_{k}^{2,3} & \beta_{k}^{2,3} & 0 \\
\vdots & \vdots & \vdots \\
\alpha_{k}^{m-1, m} & \beta_{k}^{m-1, m} & 0
\end{array}\right], } \\
& \mathbf{R}_{1, k}:= {\left[\begin{array}{ccccc}
\sigma_{r_{1,2}}^{2} & -\sigma_{r_{2}}^{2} & 0 & 0 & 0 \\
-\sigma_{r_{2}}^{2} & \sigma_{r_{2,3}}^{2} & -\sigma_{r_{3}}^{2} & 0 & 0 \\
0 & -\sigma_{r_{3}}^{2} & \sigma_{r_{3,4}}^{2} & \ddots & 0 \\
0 & 0 & \ddots & \ddots & -\sigma_{r_{m-1}}^{2} \\
0 & 0 & 0 & -\sigma_{r_{m-1}}^{2} & \sigma_{r_{m-1, m}}^{2}
\end{array}\right], } \\
& \sigma_{r_{i, j}}^{2}:=\left(1+\eta r_{i, k}\right)^{4} \sigma_{r}^{2}+\left(1+\eta r_{j, k}\right)^{4} \sigma_{r}^{2} .
\end{aligned}
$$

Based on the above analysis, the mixed linear/nonlinear system in (3) and (4) is transformed into a linear system in (3) and (11). In measurement (11), $\mathbf{B}_{k}$ is not of full column rank and the coefficient of $z_{k}$ is zero. Hence, by using the SISE algorithm for linear system (3) and (11), we could only estimate $x_{k}$ and $y_{k}$. Hence, we redefine the system equation and design a three-step unbiased minimum variance SISE algorithm. We divide the process (3) into two parts: the kinematics model in the local level plane and the kinematics model in the heave direction,

$$
\begin{aligned}
& \mathbf{X}_{1, k}=\mathbf{X}_{1, k-1}+T_{1, k-1} \mathbf{V}_{1, k-1}+w_{1, k-1}, \\
& z_{k}=z_{k-1}+t_{k-1} v_{k-1}^{z}+w_{2, k-1},
\end{aligned}
$$

where $\mathbf{X}_{1, k-1}:=\left[x_{k-1}, y_{k-1}\right]^{\mathrm{T}} \in \mathbb{R}^{2}, \mathbf{V}_{1, k-1}:=\left[v_{k-1}^{x}, v_{k-1}^{y}\right]^{\mathrm{T}} \in \mathbb{R}^{2}$, and $T_{1, k-1}:=\operatorname{diag}\left[t_{k-1}, t_{k-1}\right]$. The noises $w_{1, k-1} \in \mathbb{R}^{2}$ and $w_{2, k-1} \in \mathbb{R}$ are uncorrelated white Gaussian noise with known covariances $Q_{1, k-1}$ and $Q_{2, k-1}$. According to the measurement (11) and (4), we rewrite the measurement equation as

$$
\begin{aligned}
& \mathbf{Y}_{1, k}=\mathbf{C}_{1, k} \mathbf{X}_{1, k}+\xi_{1, k}, \\
& \mathbf{Y}_{2, k}=\mathbf{I}_{m \times 1 z k}+\xi_{2, k},
\end{aligned}
$$

$$
\mathbf{Y}_{k}=\mathbf{C}_{k} \mathbf{X}_{k}+\xi_{k}
$$

where $\mathbf{Y}_{1, k}$ is the same as $\gamma_{k}, \mathbf{Y}_{k}:=\left[\mathbf{Y}_{1, k}^{\mathrm{T}}, \mathbf{Y}_{2, k}^{\mathrm{T}}\right]^{\mathrm{T}} \in \mathbb{R}^{2 m-1}$, and $\xi_{k}:=\left[\xi_{1, k}^{\mathrm{T}}, \xi_{2, k}^{\mathrm{T}}\right]^{\mathrm{T}} \in \mathbb{R}^{2 m-1} . \mathbf{I}_{m \times 1}$ is the $m \times 1$ array of ones. $\xi_{2, k}$ is the zero mean white noise with covariance $\mathbf{R}_{2, k} \cdot \mathbf{C}_{1, k}$, $\mathbf{C}_{k}, \mathbf{R}_{2, k}$, and $\mathbf{Y}_{2, k}$ are known matrices and vector with 


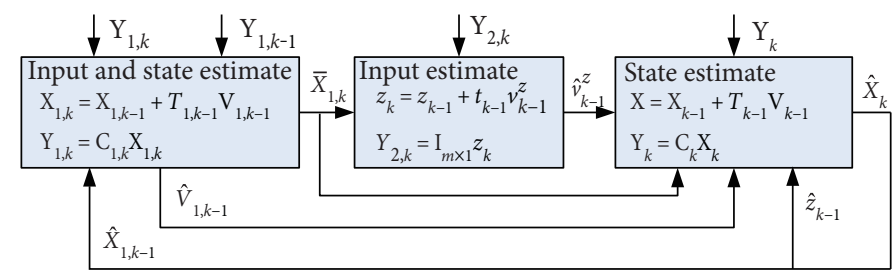

FIGURE 2: Block diagram of three-step SISE algorithm for multi-USV positioning system.

$$
\begin{aligned}
\mathbf{C}_{1, k} & :=\left[\begin{array}{cc}
\alpha_{k}^{1,2} & \beta_{k}^{1,2} \\
\alpha_{k}^{2,3} & \beta_{k}^{2,3} \\
\vdots & \vdots \\
\alpha_{k}^{m-1, m} & \beta_{k}^{m-1, m}
\end{array}\right], \\
\mathbf{C}_{k} & :=\left[\begin{array}{cc}
\mathbf{C}_{1, k} & 0 \\
0 & \mathbf{I}_{m \times 1}
\end{array}\right], \\
\mathbf{R}_{2, k} & :=\operatorname{diag}\left[\sigma_{1}^{2}, \sigma_{2}^{2}, \ldots, \sigma_{m}^{2},\right], \\
\sigma_{i}^{2} & :=\left(1+\eta r_{i, k}\right)^{2} \sigma^{2}, \\
\mathbf{Y}_{2, k} & :=\left[\varphi_{1, k}, \varphi_{2, k}, \ldots, \varphi_{m, k}\right]^{\mathrm{T}}, \\
\varphi_{i, k} & :=\left[r_{i, k}^{2}-\left(\bar{x}_{k}-x_{i, k}\right)^{2}-\left(\bar{y}_{k}-y_{i, k}\right)^{2}\right]^{1 / 2} .
\end{aligned}
$$

Note that, in (18), $\bar{x}_{k}$ and $\bar{y}_{k}$ are the position predictions of target in local level plane. Hence, in measurement (16), $z_{k}$ is the only unknown variable to be estimated. The details about them will be explained in the algorithm. As shown in Figure 2, the unbiased minimum variance SISE algorithm for the multi-USV positioning system is divided into three steps.

Step 1. For the system in (13) and (15), we design the gain matrices $\mathbf{M}_{1, k}$ and $\mathbf{K}_{1, k}$ to estimate the input $\mathbf{V}_{1, k-1}$ and predict the state $\mathbf{X}_{1, k}$.

$$
\begin{aligned}
\widehat{V}_{1, k-1}= & \mathbf{M}_{1, k}\left(\mathbf{Y}_{1, k}-\mathbf{C}_{1, k} \widehat{X}_{1, k-1}\right), \\
\bar{X}_{1, k}= & \widehat{X}_{1, k-1}+T_{1, k-1} \widehat{V}_{1, k-1} \\
& +\mathbf{K}_{1, k}\left(\mathbf{Y}_{1, k-1}-\mathbf{C}_{1, k-1} \widehat{X}_{1, k-1}\right),
\end{aligned}
$$

where $\widehat{V}_{1, k-1}$ and $\widehat{X}_{1, k-1}$ represent the estimations of $\mathbf{V}_{1, k-1}$ and $\mathbf{X}_{k-1}$ at stamp $k-1$, respectively. $\bar{X}_{1, k}:=\left[\bar{x}_{k} \bar{y}_{k}\right]$ is the predictions of $\mathbf{X}_{1, k}$ at stamp $k . \mathbf{M}_{1, k}$ and $\mathbf{K}_{1, k}$ are the gain matrices that will be designed.

Step 2. According to the predicted state $\bar{X}_{1, k}, \mathbf{Y}_{2, k}$ is calculated. For the system in (14) and (16), we design the gain matrix $\mathbf{M}_{2, k}$ to estimate the input $v_{k-1}^{z}$ and predict the state $z_{k}$.

$$
\begin{aligned}
\widehat{v}_{k-1}^{z} & =\mathbf{M}_{2, k}\left(\mathbf{Y}_{2, k}-\mathbf{I}_{m \times 1^{\hat{\imath}} k-1}\right), \\
\bar{z}_{k} & =\widehat{z}_{k-1}+t_{k-1} \widehat{v}_{k-1}^{z},
\end{aligned}
$$

where $\widehat{v}_{k-1}^{z}$ and $\widehat{z}_{k-1}$ represent the estimations of $v_{k-1}^{z}$ and $z_{k-1}$, respectively. $\bar{z}_{k}$ is the prediction of $z_{k}$. $\mathbf{M}_{2, k}$ is the gain matrix that will be determined. According to the definition of $\mathbf{V}_{1, k-1}$, we have $\mathbf{V}_{k-1}=\left[\mathbf{V}_{1, k-1}^{\mathrm{T}}, v_{k-1}^{\mathrm{Z}}{ }^{\mathrm{T}}\right]^{\mathrm{T}}$. Hence, combining the estimated inputs $\widehat{V}_{1, k-1}$ and $\widehat{v}_{k-1}^{z}$, we get the estimated input $\widehat{V}_{k-1}$.

Step 3. For the system in (3) and (17), the state $\mathbf{X}_{k}$ is estimated from the results of two previous steps.

$$
\widehat{X}_{k}=\bar{X}_{k}+\mathbf{K}_{k}\left(\mathbf{Y}_{k}-\mathbf{C}_{k} \bar{X}_{k}\right) \text {, }
$$

where $\bar{X}_{k}:=\left[\bar{X}_{1, k}^{\mathrm{T}} \bar{z}_{k}^{\mathrm{T}}\right]^{\mathrm{T}}$ is the prediction of $\mathbf{X}_{k} \cdot \mathbf{K}_{k}$ is the gain matrix that will be designed.

Note that the order of the algorithm cannot be changed. The framework of the three-step minimum variance unbiased simultaneous velocity and position estimation algorithm is illustrated in Algorithm 1. In the next two sections, we will discuss the details of this algorithm.

\section{Velocity Estimate}

Based on the above analysis, the velocity of the target is seen as the input. In this section, we establish the estimation of the unknown input $\mathbf{V}_{k-1}$. We divide the unknown input into two components, namely, the velocity in the local level plane $\mathbf{V}_{1, k-1}$ and the velocity in the heave direction $v_{k-1}^{z}$. The estimation errors of the input and state are expressed as

$$
\begin{aligned}
& \tilde{V}_{k-1}:=\mathbf{V}_{k-1}-\widehat{V}_{k-1}, \\
& \tilde{X}_{k-1}:=\mathbf{X}_{k-1}-\widehat{X}_{k-1},
\end{aligned}
$$

where $\tilde{V}_{k-1}:=\left[\tilde{V}_{1, k-1}^{\mathrm{T}}, \tilde{v}_{k-1}^{\mathrm{zT}}\right]^{\mathrm{T}}, \tilde{X}_{k-1}:=\left[\tilde{X}_{1, k-1}^{\mathrm{T}}, \tilde{z}_{k-1}^{\mathrm{T}}\right]^{\mathrm{T}}$.

3.1. Velocity Estimate in Local Level Plane. By minimizing the covariance matrix of the estimation error $\tilde{V}_{1, k-1}$, we obtain the unbiased velocity estimate in the local level plane $\widehat{V}_{1, k-1}$.

Substituting the state estimate (19) and the system (13) and (15) into the estimation error of $\mathbf{V}_{1, k-1}$, we obtain

$$
\begin{aligned}
\tilde{V}_{1, k-1}= & \mathbf{V}_{1, k-1}-\mathbf{M}_{1, k}\left(\mathbf{Y}_{1, k}-\mathbf{C}_{1, k} \widehat{X}_{1, k-1}\right) \\
= & \left(\mathbf{I}_{2}-\mathbf{M}_{1, k} \mathbf{C}_{1, k} T_{1, k-1}\right) \mathbf{V}_{1, k-1} \\
& -\mathbf{M}_{1, k}\left[\mathbf{C}_{1, k}\left(\tilde{X}_{1, k-1}+w_{1, k-1}\right)+\xi_{1, k}\right] .
\end{aligned}
$$

Since $w_{1, k-1}$ and $\xi_{1, k}$ are uncorrelated white Gaussian noises, then we have 


\section{Input:}

Positions of USVs $\left(x_{i, k}, y_{i, k}, z_{i, k}\right), i=1,2, \ldots, m$;

Position estimation $\widehat{X}_{k-1}$ and variance $P_{k-1}^{X}$;

Measurements $Y_{1, k-1}$ and $Y_{1, k}$;

Output:

Position estimation $\widehat{X}_{k}$ and velocity estimation $\widehat{V}_{1, k-1}$;

Step 1: Estimate velocity $\widehat{V}_{1, k-1}$ and predict position $\bar{X}_{1, k}$

1: $L_{1, k}=C_{1, k}\left(P_{k-1}^{X_{1}}+Q_{1, k-1}\right) C_{1, k}^{\mathrm{T}}+R_{1, k}$;

2: $M_{1, k}=\left(T_{1, k-1}^{\mathrm{T}} C_{1, k}^{\mathrm{T}} L_{1, k}^{-1} C_{1, k} T_{1, k-1}\right)^{-1} T_{1, k-1}^{\mathrm{T}} C_{1, k}^{\mathrm{T}} L_{1, k}^{-1}$;

3: $\widehat{V}_{1, k-1}=M_{1, k}\left(Y_{1, k}-C_{1, k} \widehat{X}_{1, k-1}\right)$;

4: $S_{k}=\left(I_{2}-T_{1, k-1} M_{1, k} C_{1, k}\right) P_{k-1}^{X_{1}} C_{1, k-1}^{\mathrm{T}}$;

5: $F_{k}=C_{1, k-1} P_{k-1}^{X_{1}} C_{1, k-1}^{\mathrm{T}}+R_{1, k-1}$;

6: $K_{1, k}=S_{k} F_{k}^{-1}$;

7: $\bar{X}_{1, k}=\widehat{X}_{1, k-1}+T_{1, k-1} \widehat{V}_{1, k-1}$ $+K_{1, k}\left(Y_{1, k-1}-C_{1, k-1} \widehat{X}_{1, k-1}\right) ;$

8: $P_{k \mid k-1}^{X_{1}}=\left(I_{2}-T_{1, k-1} M_{1, k} C_{1, k}\right)\left(P_{k-1}^{X_{1}}+Q_{1, k-1}\right)$ $\left(I_{2}-T_{1, k-1} M_{1, k} C_{1, k}\right)^{\mathrm{T}}$;

9: $P_{k}^{X_{1}^{*}}=P_{k \mid k-1}^{X_{1}}+T_{1, k-1} M_{1, k} R_{1, k} M_{1, k}^{\mathrm{T}} M_{1, k-1}^{\mathrm{T}}-S_{k} F_{k}^{-1} S_{k}^{\mathrm{T}}$;

Step 2: Estimate velocity $\widehat{v}_{k-1}^{z}$ and predict position $\bar{z}_{k}$

10: $H_{k}=I_{m \times 1}\left(P_{k-1}^{z}+Q_{2, k-1}\right) I_{m \times 1}^{\mathrm{T}}+R_{2, k}$;

11: $M_{2, k}=\left(t_{k-1}^{\mathrm{T}} I_{m \times 1}^{\mathrm{T}} H_{k}^{-1} I_{m \times 1} t_{k-1}\right)^{-1} t_{k-1}^{\mathrm{T}} I_{m \times 1}^{\mathrm{T}} H_{k}^{-1}$;

12: $\hat{v}_{k-1}^{z}=M_{2, k}\left(Y_{2, k}-I_{m \times 1 \hat{1}^{\overline{1}} k-1}\right)$;

13: $\bar{z}_{k}=\widehat{z}_{k-1}+t_{k-1} \widehat{v}_{k-1}^{z}$

14: $P_{k}^{z *}=\left(I-t_{k-1} M_{2-k} I_{m \times 1}\right)\left(P_{k-1}^{z}+Q_{2 k-1}\right)$

$\left(I-t_{k-1} M_{2, k} I_{m \times 1}\right)^{\mathrm{T}}+t_{k-1} M_{2, k} R_{2, k} M_{2, k}^{\mathrm{T}} t_{k-1}^{\mathrm{T}} ;$

15: $\widehat{V}_{k-1}=\left[\widehat{V}_{1, k-1}^{\mathrm{T}}\left(\widehat{v}_{k-1}^{\mathrm{x}}\right)^{\mathrm{T}}\right]^{\mathrm{T}}$;

Step 3: Estimate position $\widehat{X}_{k}$

16: $J_{k}=C_{k} P_{k}^{X *} C_{k}^{\mathrm{T}}+R_{k}, P_{k}^{X *}=\operatorname{diag}\left[P_{k}^{X_{1}^{*}}, P_{k}^{z *}\right]$;

17: $P_{k}^{X *}=\operatorname{diag}\left[P_{k}^{X_{1}^{*}}, P_{k}^{z *}\right]$;

18: $K_{k}=P_{k}^{X *} C_{k}^{\mathrm{T}} J_{k}^{-1}$;

19: $\widehat{X}_{k}=\bar{X}_{k}+K_{k}\left(Y_{k}-C_{k} \bar{X}_{k}\right)$;

20: $P_{k}^{X}=P_{k}^{X *}-P_{k}^{X *} C_{k}^{\mathrm{T}}\left(C_{k} P_{k}^{X *} C_{k}^{\mathrm{T}}+R_{k}\right)^{-1} C_{k} P_{k}^{X *}$;

Return Position estimation $\widehat{X}_{k}$ and variance $P_{k}^{X}$;

Algorithm 1: Framework of three-step SISE algorithm.

$$
\begin{aligned}
\mathbb{E}\left(\tilde{V}_{1, k-1}\right)= & \left(\mathbf{I}_{2}-\mathbf{M}_{1, k} \mathbf{C}_{1, k} T_{1, k-1}\right) \mathbb{E}\left(\mathbf{V}_{1, k-1}\right) \\
& -\mathbf{M}_{1, k} \mathbf{C}_{1, k} \mathbb{E}\left(\tilde{X}_{1, k-1}\right) .
\end{aligned}
$$

In MLBL, $\mathbb{E}\left(\mathbf{V}_{1, k-1}\right)$ is not equal to zero, and it changes with time. Hence, for the system in (13) and (15), $\widehat{V}_{1, k-1}$ is an unbiased estimator of $\mathbf{V}_{1, k-1}$ if and only if the following conditions are satisfied.

$$
\begin{aligned}
\mathbb{E}\left(\tilde{X}_{1, k-1}\right) & =0, \\
\mathbf{I}_{2} & =\mathbf{M}_{1, k} \mathbf{C}_{1, k} T_{1, k-1} .
\end{aligned}
$$

Theorem 1. Let $\widehat{V}_{1, k-1}$ is an unbiased estimator and $\mathbf{M}_{1, k}$ is given by

$$
\mathbf{M}_{1, k}=\left(T_{1, k-1}^{\mathrm{T}} \mathbf{C}_{1, k}^{\mathrm{T}} L_{1, k}^{-1} \mathbf{C}_{1, k} T_{1, k-1}\right)^{-1} T_{1, k-1}^{\mathrm{T}} \mathbf{C}_{1, k}^{\mathrm{T}} L_{1, k}^{-1},
$$

where $L_{1, k}=\mathbf{C}_{1, k}\left(\mathbf{P}_{k-1}^{X_{1}}+Q_{1, k-1}\right) \mathbf{C}_{1, k}^{\mathrm{T}}+\mathbf{R}_{1, k}$, then (19) is the MVUE of $\mathbf{V}_{1, k-1}$.

Proof. Under the unbiasedness (27), the estimation error of $\mathbf{V}_{1, k-1}$ is simplified as

$$
\tilde{V}_{1, k-1}=-\mathbf{M}_{1, k}\left[\mathbf{C}_{1, k}\left(\tilde{X}_{1, k-1}+w_{1, k-1}\right)+\xi_{1, k}\right] .
$$

Substituting (29) into the covariance matrix ${ }_{s}$, then we have

$$
\mathbf{P}_{k-1}^{V_{1}}=\mathbf{M}_{1, k}\left[\mathbf{C}_{1, k}\left(\mathbf{P}_{k-1}^{X_{1}}+Q_{1, k-1}\right) \mathbf{C}_{1, k}^{\mathrm{T}}+\mathbf{R}_{1, k}\right] \mathbf{M}_{1, k}^{\mathrm{T}} .
$$

For the minimum variance estimation, the problem is equivalent to finding the gain matrix $\mathbf{M}_{1, k}$ which minimizes the trace of (30) subject to (27). The Lagrangian is [41] 


$$
\begin{aligned}
\operatorname{tr}\left(\mathbf{P}_{k-1}^{V_{1}}\left(\boldsymbol{\lambda}_{k-1}\right)\right)= & \operatorname{tr}\left(\mathbf{M}_{1, k}\left[\mathbf{C}_{1, k}\left(\mathbf{P}_{k-1}^{X_{1}}+Q_{1, k-1}\right) \mathbf{C}_{1, k}^{\mathrm{T}}+\mathbf{R}_{1, k}\right] \mathbf{M}_{1, k}^{\mathrm{T}}\right) \\
& -2 \operatorname{tr}\left(\left(\mathbf{I}_{2}-\mathbf{M}_{1, k} \mathbf{C}_{1, k} T_{1, k-1}\right) \boldsymbol{\lambda}_{k-1}^{\mathrm{T}}\right),
\end{aligned}
$$

where $\boldsymbol{\lambda}_{k-1}$ is the matrix of Lagrange multipliers. The minimum value of $\operatorname{tr}\left(\mathbf{P}_{k-1}^{V}\left(\boldsymbol{\lambda}_{k-1}\right)\right)$ is reached when the following equation is satisfied.

$$
2\left[\mathbf{C}_{1, k}\left(\mathbf{P}_{k-1}^{X}+Q_{1, k-1}\right) \mathbf{C}_{1, k}^{\mathrm{T}}+\mathbf{R}_{1, k}\right] \mathbf{M}_{1, k}^{\mathrm{T}}-2 \mathbf{C}_{1, k} T_{1, k-1} \boldsymbol{\lambda}_{k-1}^{\mathrm{T}}=0 .
$$

Substituting (27) into (32), we have

$$
\boldsymbol{\lambda}_{k-1}=\left(T_{1, k-1}^{\mathrm{T}} \mathbf{C}_{1, k}^{\mathrm{T}} L_{1, k}^{-1} \mathbf{C}_{1, k} T_{1, k-1}\right)^{-1} .
$$

Define $l_{1, k}=\mathbf{C}_{1, k}\left(\tilde{X}_{1, k-1}+w_{1, k-1}\right)+\xi_{1, k}$, then we have $L_{1, k}=\mathbb{E}\left(l_{1, k} l_{1, k}^{\mathrm{T}}\right)$. It is obvious that $L_{1, k}$ is positive definite and reversible. Combining (32) and (33), we obtain the gain matrix (28).

From Theorem 1, we get the gain matrix $\mathbf{M}_{1, k}$ and prove that (19) is the MVUE of $\mathbf{V}_{1, k-1}$.

3.2. Velocity Estimate in Heave Direction. The velocity estimator in the heave direction is shown as (21). We estimate the velocity $v_{k-1}^{z}$ in two stages. Firstly, according to (20), we predict the position of the target in the local level plane $\bar{X}_{1, k}$. Then, based on the predicted position $\bar{X}_{1, k}$, we estimate the velocity in the heave direction $\widehat{v}_{k-1}^{z}$. In this section, we will discuss the unbiased property of $\widehat{v}_{k-1}^{z}$ and design the gain matrix $\mathbf{M}_{2, k}$.

Similar to the velocity estimate in local level plane, by minimizing the covariance matrix of the estimation error $\tilde{v}_{k-1}^{z}$, we get the unbiased velocity estimate in heave direction. The following conclusions are obtained.

For the system in (14) and (16), $\widehat{v}_{k-1}^{z}$ is an unbiased estimator of $v_{k-1}^{z}$ if and only if the following conditions are satisfied.

$$
\begin{aligned}
\mathbb{E}\left(\tilde{z}_{k-1}\right) & =0, \\
\mathbf{I}_{1} & =\mathbf{M}_{2, k} \mathbf{I}_{m \times 1} t_{k-1} .
\end{aligned}
$$

Let $\widehat{v}_{k-1}^{z}$ is an unbiased estimator and $\mathbf{M}_{2, k}$ is given by

$$
\mathbf{M}_{2, k}=\left(t_{k-1}^{\mathrm{T}} \mathbf{I}_{m \times 1}^{\mathrm{T}} H_{k}^{-1} \mathbf{I}_{m \times 1} t_{k-1}\right)^{-1} t_{k-1}^{\mathrm{T}} \mathbf{I}_{m \times 1}^{\mathrm{T}} H_{k}^{-1},
$$

where $H_{k}=\mathbf{I}_{m \times 1}\left(\mathbf{P}_{k-1}^{z}+Q_{2, k-1}\right) \mathbf{I}_{m \times 1}^{\mathrm{T}}+\mathbf{R}_{2, k}$, then (21) is the MVUE of $v_{k-1}^{z}$.

Note that under the unbiasedness (34), (21) is simplified as

$$
\tilde{v}_{k-1}^{z}=-\mathbf{M}_{2, k}\left[\mathbf{I}_{m \times 1}\left(\tilde{z}_{k-1}+w_{2, k-1}\right)+\xi_{2, k}\right] .
$$

\section{Position Estimate}

In Section 3, we estimated the velocity of the target. In this section, we will estimate the position of the target. As was mentioned above, the position of the target is seen as the state. First, we predict the states $\bar{X}_{1, k}$ and $\bar{z}_{k}$ and then estimate the minimum variance unbiased state $\widehat{X}_{k}$.

4.1. Position Predict. The position predictors are shown as (20) and (22). Based on the estimated velocity in the heave direction $\widehat{v}_{k-1}^{z}$, we can easily predict the target position in the heave direction. In order to predict the target position in the local level plane, we will design the gain matrix $\mathbf{K}_{1, k}$ in this section. The unbiased properties of $\bar{z}_{k}$ and $\bar{X}_{1, k}$ are also analysed.

Define $\tilde{z}_{k}^{*}=z_{k}-\bar{z}_{k}$ as the prediction error of $z_{k}$. From (14) and (22), we obtain

$$
\tilde{z}_{k}^{*}=\tilde{z}_{k-1}+t_{k-1} \tilde{v}_{k-1}^{z}+w_{2, k-1} .
$$

Since $\mathbb{E}\left(w_{2, k-1}\right)=0$, then we have

$$
\mathbb{E}\left(\tilde{z}_{k}^{*}\right)=\mathbb{E}\left(\tilde{z}_{k-1}\right)+t_{k-1} \mathbb{E}\left(\tilde{v}_{k-1}^{z}\right) .
$$

Then, for the system in (14), $\bar{z}_{k}$ is an unbiased estimator of $z_{k}$ if and only if the following conditions are satisfied.

$$
\begin{aligned}
& \mathbb{E}\left(\tilde{z}_{k-1}\right)=0, \\
& \mathbb{E}\left(\tilde{v}_{k-1}^{z}\right)=0 .
\end{aligned}
$$

Define $\tilde{X}_{1, k}^{*}=\mathbf{X}_{1, k}-\bar{X}_{1, k}$ as the prediction error of $\mathbf{X}_{1, k}$. Substituting the estimator (20) and the system (13) and (15) into this prediction error, we obtain

$$
\begin{aligned}
\tilde{X}_{1, k}^{*}= & \mathbf{X}_{1, k}-\widehat{X}_{1, k-1}-T_{1, k-1} \widehat{V}_{1, k-1} \\
& -\mathbf{K}_{1, k}\left(\mathbf{Y}_{1, k-1}-\mathbf{C}_{1, k-1} \widehat{X}_{1, k-1}\right) \\
= & \left(\mathbf{I}_{2}-\mathbf{K}_{1, k} \mathbf{C}_{1, k}\right) \tilde{X}_{1, k-1}+T_{1, k-1} \tilde{V}_{1, k-1} \\
& +w_{1, k-1}-\mathbf{K}_{1, k} \xi_{1, k-1} .
\end{aligned}
$$

Since $w_{1, k-1}$ and $\xi_{1, k-1}$ are uncorrelated white Gaussian noises, then the prediction error of $\mathbf{X}_{1, k}$ is simplified as

$$
\mathbb{E}\left(\tilde{X}_{1, k}^{*}\right)=\left(\mathbf{I}_{2}-\mathbf{K}_{1, k} \mathbf{C}_{1, k}\right) \mathbb{E}\left(\tilde{X}_{1, k-1}\right)+T_{1, k-1} \mathbb{E}\left(\tilde{V}_{1, k-1}\right) .
$$

It is obviously that, for the system in (13) and (15), $\bar{X}_{1, k}$ is an unbiased estimator of $\mathbf{X}_{1, k}$ if and only if the following conditions are satisfied.

$$
\begin{aligned}
& \mathbb{E}\left(\tilde{X}_{1, k-1}\right)=0, \\
& \mathbb{E}\left(\tilde{V}_{1, k-1}\right)=0 .
\end{aligned}
$$

Theorem 2. Let $\bar{X}_{1, k}$ is an unbiased estimator and the gain matrix $\mathbf{K}_{1, k}$ is given by

$$
\mathbf{K}_{1, k}=S_{k} F_{k}^{-1},
$$

where $S_{k}=\left(\mathbf{I}_{2}-T_{1, k-1} \mathbf{M}_{1, k} \mathbf{C}_{1, k}\right) \mathbf{P}_{k-1}^{X_{1}} \mathbf{C}_{1, k-1}^{\mathrm{T}}$ and $F_{k}=\mathbf{C}_{1, k-1}$ $\mathbf{P}_{k-1}^{X_{1}} \mathbf{C}_{1, k-1}^{\mathrm{T}}+\mathbf{R}_{1, k-1}$, then (20) is the MVUE of $\mathbf{X}_{1, k}$. The covariance matrix of the prediction error $\tilde{X}_{1, k}^{*}$ is

$$
\mathbf{P}_{k}^{X_{1}^{*}}=\mathbf{P}_{k \mid k-1}^{X_{1}}+T_{1, k-1} \mathbf{M}_{1, k} \mathbf{R}_{1, k} \mathbf{M}_{1, k}^{\mathrm{T}} T_{1, k-1}^{\mathrm{T}}-S_{k} F_{k}^{-1} S_{k}^{\mathrm{T}},
$$

where 


$$
\begin{aligned}
\mathbf{P}_{k \mid k-1}^{X_{1}}= & \left(\mathbf{I}_{2}-T_{1, k-1} \mathbf{M}_{1, k} \mathbf{C}_{1, k}\right)\left(\mathbf{P}_{k-1}^{X_{1}}+Q_{1, k-1}\right) \\
& \cdot\left(\mathbf{I}_{2}-T_{1, k-1} \mathbf{M}_{1, k} \mathbf{C}_{1, k}\right)^{\mathrm{T}} .
\end{aligned}
$$

Proof. Substituting the estimation error (29) into (40), the prediction error $\tilde{X}_{1, k}^{*}$ is simplified as

$$
\begin{aligned}
\tilde{X}_{1, k}^{*}= & \left(\mathbf{I}_{2}-T_{1, k-1} \mathbf{M}_{1, k} \mathbf{C}_{1, k}\right)\left(\tilde{X}_{1, k-1}+w_{1, k-1}\right) \\
& -\mathbf{K}_{1, k} \mathbf{C}_{1, k-1} \tilde{X}_{1, k-1}-\mathbf{K}_{1, k} \xi_{1, k-1}-T_{1, k-1} \mathbf{M}_{1, k} \xi_{1, k} .
\end{aligned}
$$

Then, the covariance matrix $\mathbf{P}_{k}^{X_{1}^{*}}:=\mathbb{E}\left(\tilde{X}_{1, k}^{*} \tilde{X}_{1, k}^{* \mathrm{~T}}\right)$ is written as

$$
\begin{aligned}
\mathbf{P}_{k}^{X_{1}^{*}}= & \mathbf{K}_{1, k} F_{k} \mathbf{K}_{1, k}^{\mathrm{T}}-\mathbf{K}_{1, k} S_{k}^{\mathrm{T}}-S_{k} \mathbf{K}_{1, k}^{\mathrm{T}}+\mathbf{P}_{k \mid k-1}^{X_{1}} \\
& +T_{1, k-1} \mathbf{M}_{1, k} \mathbf{R}_{1, k} \mathbf{M}_{1, k}^{\mathrm{T}} T_{1, k-1}^{\mathrm{T}},
\end{aligned}
$$

where $\mathbf{P}_{k-1}^{X_{1}}:=\mathbb{E}\left(\tilde{X}_{1, k-1} \tilde{X}_{1, k-1}^{\mathrm{T}}\right)$. Taking the derivatives with respect to the gain matrix $\mathbf{K}_{1, k}$ equal to zero yields

$$
2 F_{k} \mathbf{K}_{1, k}^{\mathrm{T}}-2 S_{k}^{\mathrm{T}}=0 .
$$

Define $f_{k}=\mathbf{C}_{1, k-1} \tilde{X}_{1, k-1}+\xi_{1, k-1}$, then we have $F_{k}=$ $\mathbb{E}\left(f_{k} f_{k}^{\mathrm{T}}\right)$. It is obvious that $F_{k}$ is positive definite and reversible. Based on the above analysis, we get (43). Substituting (43) into (47), we obtain the covariance matrix (44).

4.2. Position Estimate. In Section 4.1, we predicted the position of the target. In this section, we will estimate the position of the target by using the estimated velocity and the predicted position. First, we discuss the unbiased property of $\widehat{X}_{k}$ and then design the gain matrix $\mathbf{K}_{k}$.

Define $\tilde{X}_{k}^{*}:=\mathbf{X}_{k}-\bar{X}_{k}$ and $\tilde{X}_{k}:=\mathbf{X}_{k}-\widehat{X}_{k}$ as the prediction and estimation errors of $\mathbf{X}_{k}$, respectively. From (17) and (23), we obtain

$$
\begin{aligned}
\tilde{X}_{k} & =\mathbf{X}_{k}-\bar{X}_{k}-\mathbf{K}_{k}\left(\mathbf{C}_{k} \mathbf{X}_{k}+\xi_{k}-\mathbf{C}_{k} \bar{X}_{k}\right) \\
& =\left(\mathbf{I}_{3}-\mathbf{K}_{k} \mathbf{C}_{k}\right) \tilde{X}_{k}^{*}-\mathbf{K}_{k} \xi_{k},
\end{aligned}
$$

where $\mathbf{I}_{3}$ is the $3 \times 3$ identity matrix. Since $\mathbb{E}\left(\xi_{k}\right)=0$, then we have

$$
\mathbb{E}\left(\tilde{X}_{k}\right)=\left(\mathbf{I}_{3}-\mathbf{K}_{k} \mathbf{C}_{k}\right) \mathbb{E}\left(\tilde{X}_{k}^{*}\right) .
$$

By definition, we have

$$
\mathbb{E}\left(\tilde{X}_{k}^{*}\right)=\left[\begin{array}{c}
\mathbb{E}\left(\tilde{X}_{1, k}^{*}\right) \\
\mathbb{E}\left(\tilde{z}_{k}^{*}\right)
\end{array}\right] .
$$

It can be seen that $\widehat{X}_{k}$ is an unbiased estimator if and only if the following equations are satisfied.

$$
\begin{aligned}
\mathbb{E}\left(\tilde{X}_{1, k}^{*}\right) & =0, \\
\mathbb{E}\left(\tilde{z}_{k}^{*}\right) & =0 .
\end{aligned}
$$

TABLE 1: Simulation parameters.

\begin{tabular}{lccc}
\hline $\mathbf{X}_{0}$ & $\mathbf{P}_{0}$ & $Q_{1}$ & $Q_{2}$ \\
\hline$(0,0,300)$ & $\operatorname{diag}\left[10^{3}, 10^{3}, 10^{3}\right]$ & $\operatorname{diag}[10,10]$ & 10 \\
\hline
\end{tabular}

TABle 2: Parameters of USVs and target in optimal formation.

\begin{tabular}{lcccc}
\hline Vehicle & $\begin{array}{c}\text { Initial position } \\
(\mathrm{m})\end{array}$ & $\begin{array}{c}\text { Forward speed } \\
(\mathrm{knots})\end{array}$ & $\begin{array}{c}\text { Heading } \\
\left({ }^{\circ}\right)\end{array}$ & $\begin{array}{c}\text { Pitch } \\
\left({ }^{\circ}\right)\end{array}$ \\
\hline Target & $(0,0,300)$ & 2.0003 & $90-0.5 \mathrm{t}$ & 1 \\
USV1 & $(400,0,0)$ & 2 & $90-0.5 \mathrm{t}$ & 0 \\
USV2 & $(0,400,0)$ & 2 & $90-0.5 \mathrm{t}$ & 0 \\
USV3 & $(-400,0,0)$ & 2 & $90-0.5 \mathrm{t}$ & 0 \\
USV3 & $(0,-400,0)$ & 2 & $90-0.5 \mathrm{t}$ & 0 \\
\hline
\end{tabular}

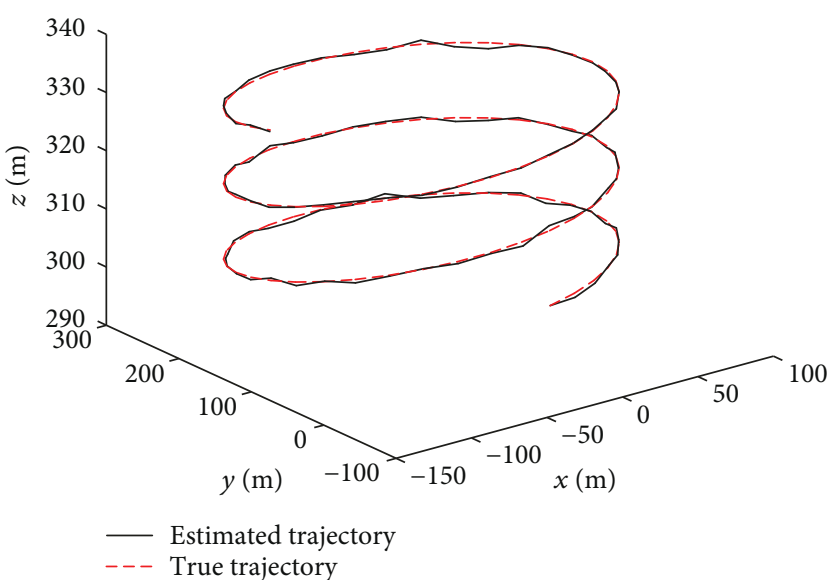

Figure 3: True and estimated trajectories of the target in optimal formation.

Based on the results in Section 3, we get that $\widehat{X}_{k}$ is an unbiased estimator of $\mathbf{X}_{k}$ if and only if the following conditions are satisfied.

$$
\begin{aligned}
& \mathbb{E}\left(\tilde{X}_{k-1}\right)=0, \\
& \mathbb{E}\left(\tilde{V}_{k-1}\right)=0 .
\end{aligned}
$$

Theorem 3. Let $\widehat{X}_{k}$ is an unbiased estimator and the gain matrix $\mathbf{K}_{k}$ is given by

$$
\mathbf{K}_{k}=\mathbf{P}_{k}^{X *} \mathbf{C}_{k}^{\mathrm{T}} J_{k}^{-1},
$$

where $J_{k}=\mathbf{C}_{k} \mathbf{P}_{k}^{X *} \mathbf{C}_{k}^{\mathrm{T}}+\mathbf{R}_{k}, \mathbf{P}_{k}^{X *}=\operatorname{diag}\left[\mathbf{P}_{k}^{X_{1}^{*}}, \mathbf{P}_{k}^{z *}\right], \quad \mathbf{P}_{k}^{z *}=t_{k-1}$ $\mathbf{M}_{2, k} \mathbf{R}_{2, k} \mathbf{M}_{2, k}^{\mathrm{T}} t_{k-1}^{\mathrm{T}}+t_{k}\left(\mathbf{P}_{k-1}^{z}+Q_{2, k-1}\right) t_{k}^{\mathrm{T}}$, and $t_{k}=\mathbf{I}-t_{k-1} \mathbf{M}_{2, k}$ $\mathbf{I}_{m \times 1}$, then (23) is the MVUE of $\mathbf{X}_{k}$. The covariance matrix of the estimation error $\tilde{X}_{k}$ is

$$
\mathbf{P}_{k}^{X}=\mathbf{P}_{k}^{X *}-\mathbf{P}_{k}^{X *} \mathbf{C}_{k}^{\mathrm{T}}\left(\mathbf{C}_{k} \mathbf{P}_{k}^{X *} \mathbf{C}_{k}^{\mathrm{T}}+\mathbf{R}_{k}\right)^{-1} \mathbf{C}_{k} \mathbf{P}_{k}^{X *} .
$$

Proof. Substituting (37) and (40) into (49), the estimation error of $\mathbf{X}_{k}$ can be written as 

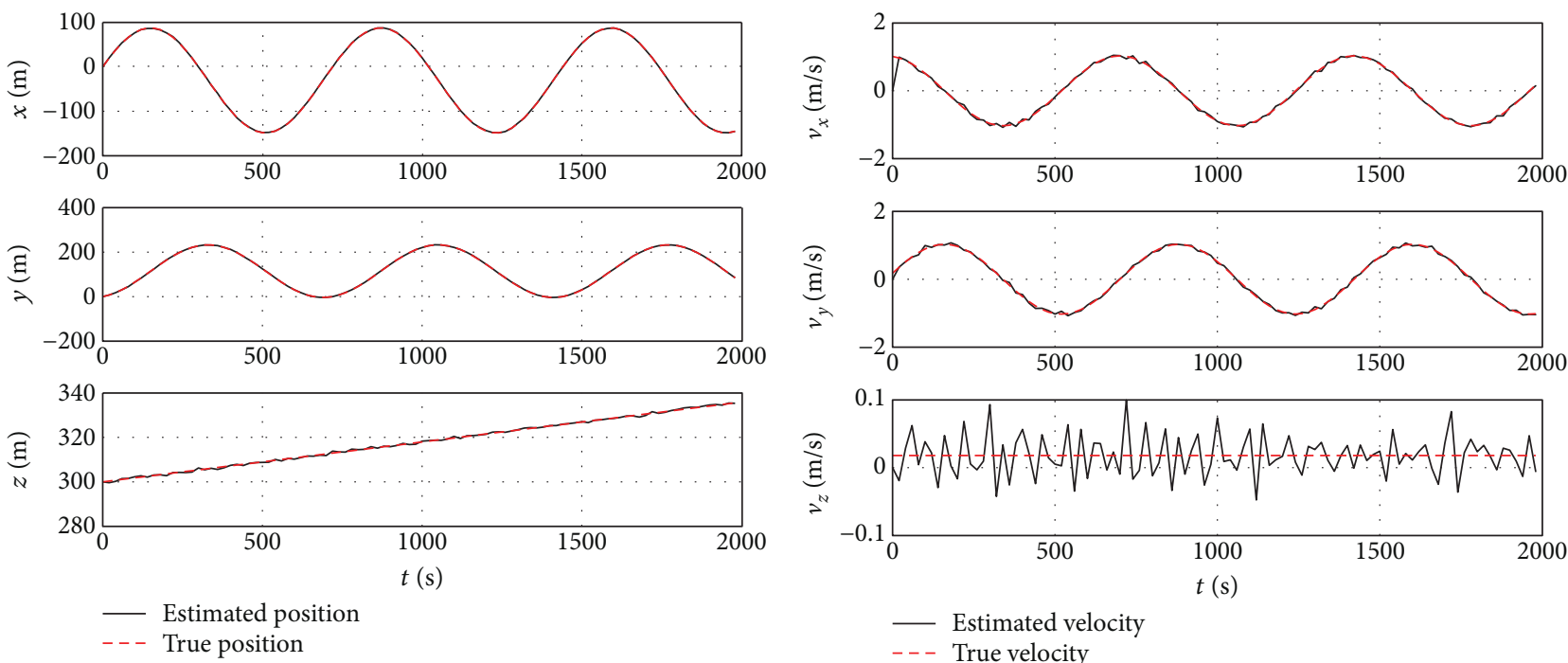

(a)

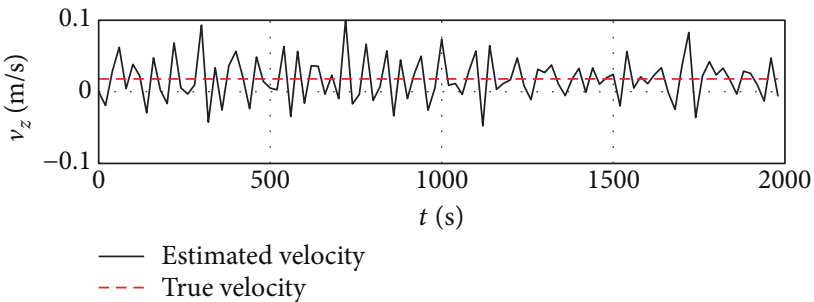

(b)

FIgURE 4: Comparison of the true and estimated values in optimal formation. (a) True and estimated position. (b) True and estimated velocity.

$$
\tilde{X}_{k}=\left(\mathbf{I}_{3}-\mathbf{K}_{k} \mathbf{C}_{k}\right)\left[\begin{array}{c}
\tilde{X}_{1, k}^{*} \\
\tilde{z}_{k}^{*}
\end{array}\right]-\mathbf{K}_{k} \xi_{k},
$$

with

$$
\begin{aligned}
\tilde{X}_{1, k}^{*}= & \left(\mathbf{I}_{2}-\mathbf{K}_{1, k} \mathbf{C}_{1, k}\right) \tilde{X}_{1, k-1}+T_{1, k-1} \tilde{V}_{1, k-1} \\
& +w_{1, k-1}-\mathbf{K}_{1, k} \xi_{1, k-1}, \\
\tilde{z}_{k}^{*}= & \tilde{z}_{k-1}+t_{k-1} \tilde{v}_{k-1}^{z}+w_{2, k-1} .
\end{aligned}
$$

From (56), (57), and (58), $\xi_{k}$ is unrelated with $\tilde{X}_{1, k}^{*}$ and $\tilde{z}_{k}^{*}$. Then, the covariance matrix $\mathbf{P}_{k}^{X}:=\mathbb{E}\left(\tilde{X}_{k} \tilde{X}_{k}^{\mathrm{T}}\right)$ is written as

$$
\mathbf{P}_{k}^{X}=\mathbf{P}_{k}^{X *}-\mathbf{K}_{k} \mathbf{C}_{k} \mathbf{P}_{k}^{X *}-\mathbf{P}_{k}^{X *} \mathbf{C}_{k}^{\mathrm{T}} \mathbf{K}_{k}^{\mathrm{T}}+\mathbf{K}_{k} J_{k} \mathbf{K}_{k}^{X},
$$

where

$$
\begin{aligned}
& \mathbf{P}_{k}^{X *}= {\left[\begin{array}{cc}
\mathbb{E}\left(\tilde{X}_{1, k}^{*} \tilde{X}_{1, k}^{* \mathrm{~T}}\right) & \mathbb{E}\left(\tilde{X}_{1, k}^{*} \tilde{z}_{k}^{* \mathrm{~T}}\right) \\
\mathbb{E}\left(\tilde{X}_{1, k}^{*} \tilde{z}_{k}^{* \mathrm{~T}}\right)^{\mathrm{T}} & \mathbb{E}\left(\tilde{z}_{k}^{*} \tilde{z}_{k}^{* \mathrm{~T}}\right)
\end{array}\right] } \\
&:=\left[\begin{array}{cc}
\mathbf{P}_{k}^{X_{1}^{*}} & \mathbf{P}_{k}^{X_{1}^{*}, z *} \\
\left(\mathbf{P}_{k}^{X_{1}^{*}, z *}\right)^{\mathrm{T}} & \mathbf{P}_{k}^{z *}
\end{array}\right] .
\end{aligned}
$$

Substituting (36) into (37), the prediction error of $z_{k}$ is written as

$$
\tilde{z}_{k}^{*}=\left(\mathbf{I}-t_{k-1} \mathbf{M}_{2, k} \mathbf{I}_{m \times 1}\right)\left(\tilde{z}_{k-1}-w_{2, k-1}\right)-t_{k-1} \mathbf{M}_{2, k} \xi_{2, k} .
$$

It follows that

$$
\begin{aligned}
\mathbf{P}_{k}^{z *}= & \left(\mathbf{I}-t_{k-1} \mathbf{M}_{2, k} \mathbf{I}_{m \times 1}\right)\left(\mathbf{P}_{k-1}^{z}+Q_{2, k-1}\right)\left(\mathbf{I}-t_{k-1} \mathbf{M}_{2, k} \mathbf{I}_{m \times 1}\right)^{\mathrm{T}} \\
& +t_{k-1} \mathbf{M}_{2, k} \mathbf{R}_{2, k} \mathbf{M}_{2, k}^{\mathrm{T}} t_{k-1}^{\mathrm{T}},
\end{aligned}
$$

where $\mathbf{P}_{k-1}^{z}:=\mathbb{E}\left(\tilde{z}_{k-1} \tilde{z}_{k-1}^{\mathrm{T}}\right)$. The prediction errors $\tilde{X}_{1, k}^{*}$ and $\tilde{z}_{k}^{*}$ are shown in (46) and (61). It is obvious that $\tilde{X}_{1, k}^{*}$ and $\tilde{z}_{k}^{*}$ are independent, then we have $\mathbf{P}_{k}^{X_{1}^{*}, z^{*}}=0$. Taking the derivatives of (59) with respect to the gain matrix $\mathbf{K}_{k}$ equal to zero yields

$$
-2 \mathbf{P}_{k}^{X *} C_{k}^{\mathrm{T}}+2 \mathbf{K}_{k} J_{k}=0
$$

Define $j_{k}=\mathbf{C}_{k} \tilde{X}_{k}^{*}+\xi_{k}$, we have $J_{k}=\mathbb{E}\left(j_{k} j_{k}^{\mathrm{T}}\right)$. Since $J_{k}$ is positive definite and reversible, we obtain the gain matrix (54). Substituting (54) into (59), we get the covariance matrix (55).

\section{Estimation Conditions}

In Sections 3 and 4, we have derived the necessary and sufficient conditions for unbiased estimators $\widehat{V}_{1, k-1}, \widehat{v}_{k-1}^{z}$, and $\widehat{X}_{k}$, respectively. In this section, we will analyse the corresponding conditions in multi-USV positioning system.

From the results about unbiased velocity estimates in Sections 3.1 and 3.2, we obtain that $\widehat{V}_{k-1}$ is an unbiased estimator if and only if the following conditions are satisfied.

$$
\begin{aligned}
\mathbb{E}\left(\tilde{X}_{k-1}\right) & =0, \\
\mathbf{I}_{3} & =\mathbf{M}_{k} \mathbf{C}_{k} T_{k-1} .
\end{aligned}
$$

Combining it with the unbiased result in Section 4.2, we obtain that $\widehat{X}_{k}$ and $\widehat{V}_{k-1}$ are unbiased estimators if (64) and (65) are satisfied. By inductive arguments, for the system in 

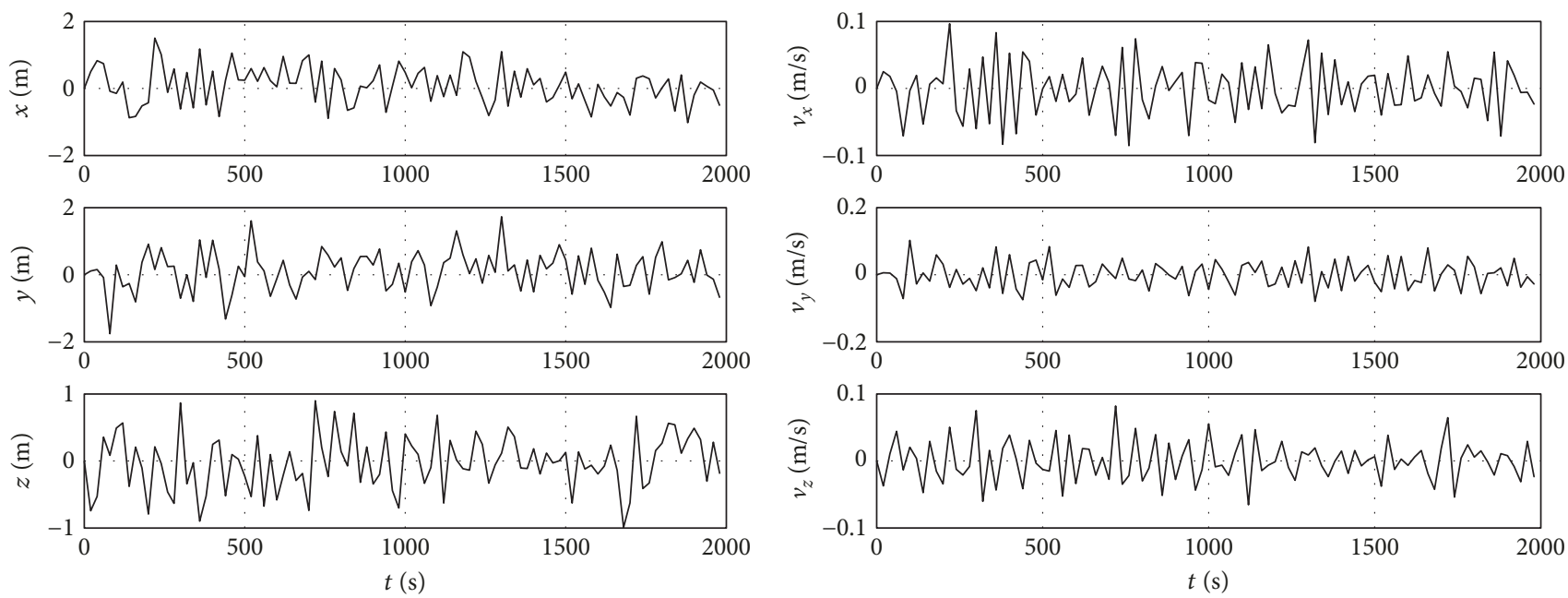

(a)

(b)

FIGURE 5: Estimation error in optimal formation. (a) Estimation error of position. (b) Estimation error of velocity.

TABLE 3: Parameters of USVs and target in general formation.

\begin{tabular}{lcccc}
\hline Vehicle & $\begin{array}{c}\text { Initial position } \\
(\mathrm{m})\end{array}$ & $\begin{array}{c}\text { Forward speed } \\
\text { (knots) }\end{array}$ & Heading $\left({ }^{\circ}\right)$ & Pitch $\left(^{\circ}\right)$ \\
\hline USV1 & $(400,0,0)$ & 3 & $90-0.25 \mathrm{t}$ & 0 \\
USV2 & $(0,400,0)$ & 1.5 & 90 & 0 \\
USV3 & $(-400,0,0)$ & 1 & $90-2 \mathrm{t}$ & 0 \\
USV4 & $(0,-400,0)$ & 2 & $90-0.5 \mathrm{t}$ & 0 \\
\hline
\end{tabular}

(3) and (17), $\widehat{X}_{k}$ and $\widehat{V}_{k-1}$ are unbiased estimators if and only if the following conditions are satisfied.

$$
\begin{aligned}
\mathbb{E}\left(\tilde{X}_{0}\right) & =0, \\
\mathbf{I}_{3} & =\mathbf{M}_{k} \mathbf{C}_{k} T_{k-1},
\end{aligned}
$$

where $\mathbf{M}_{k}=\operatorname{diag}\left[\mathbf{M}_{1, k}, \mathbf{M}_{2, k}\right]$.

From (65), we get that $\mathbf{C}_{k} T_{k-1}$ is a full column rank. The following constraint should be satisfied.

$$
\operatorname{rank}\left(\mathbf{C}_{k} T_{k-1}\right)=\operatorname{rank}\left(\mathbf{C}_{k}\right)=3 .
$$

It follows that

$$
\operatorname{rank}\left(\mathbf{C}_{1, k}\right)=\operatorname{rank}\left(\left[\begin{array}{cc}
x_{p, k}-x_{1, k} & y_{p, k}-y_{1, k} \\
x_{p, k}-x_{2, k} & y_{p, k}-y_{2, k} \\
\vdots & \vdots \\
x_{p, k}-x_{m, k} & y_{p, k}-y_{m, k}
\end{array}\right]\right)=2 \text {. }
$$

Hence, the following equation should be satisfied.

$$
\frac{x_{p, k}-x_{q, k}}{x_{p, k}-x_{r, k}} \neq \frac{y_{p, k}-y_{q, k}}{y_{p, k}-y_{r, k}},
$$

where $p, q, r \in\{1,2, \ldots, m\}$. That is to say, two conditions should be satisfied: at least three USVs are required to locate the target, these three USVs should be noncollinear.

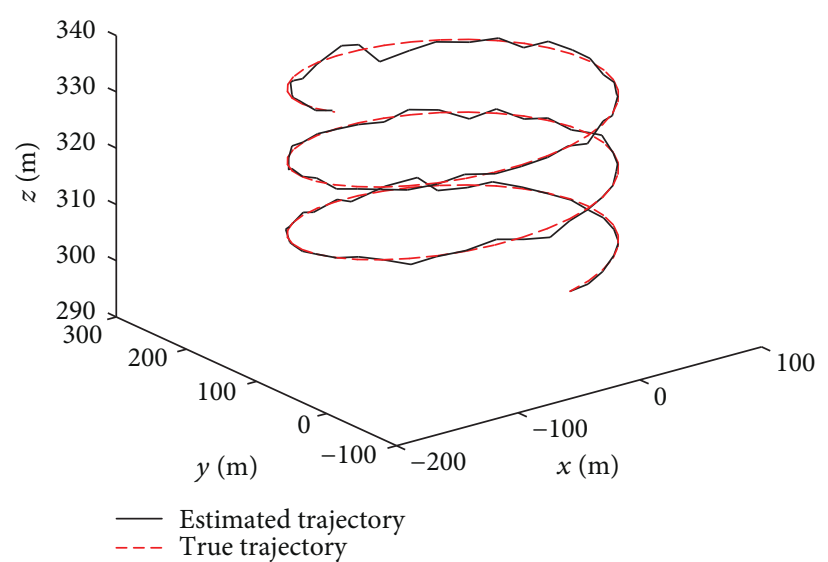

FIgURE 6: True and estimated trajectories of the target in general formation.

\section{Simulations}

In this section, we illustrate the effectiveness of the problem formulation and demonstrate the performance of the proposed algorithm. We assume that the MLBL system consists of four USVs. The system runs at the sampling period of $t_{k}=20 \mathrm{~s}$. We choose the parameters of the range measurement error as $\eta=0.001 \mathrm{~m}^{-1}$ and $\sigma_{r}=1 \mathrm{~m}$. The positioning error of USV $i\left(\varepsilon_{x_{i, k}}, \varepsilon_{y_{i, k}}\right)$ follows standardized normal distribution. Table 1 shows the parameters used in the algorithm.

The matrices of the time-variant, discrete-time system are described by

$$
\begin{aligned}
T_{k}= & \operatorname{diag}[20,20,20], \\
\mathbf{C}_{1, k}:= & {\left[\begin{array}{cc}
\alpha_{k}^{1,2} & \beta_{k}^{1,2} \\
\alpha_{k}^{2,3} & \beta_{k}^{2,3} \\
\alpha_{k}^{3,4} & \beta_{k}^{3,4}
\end{array}\right], }
\end{aligned}
$$



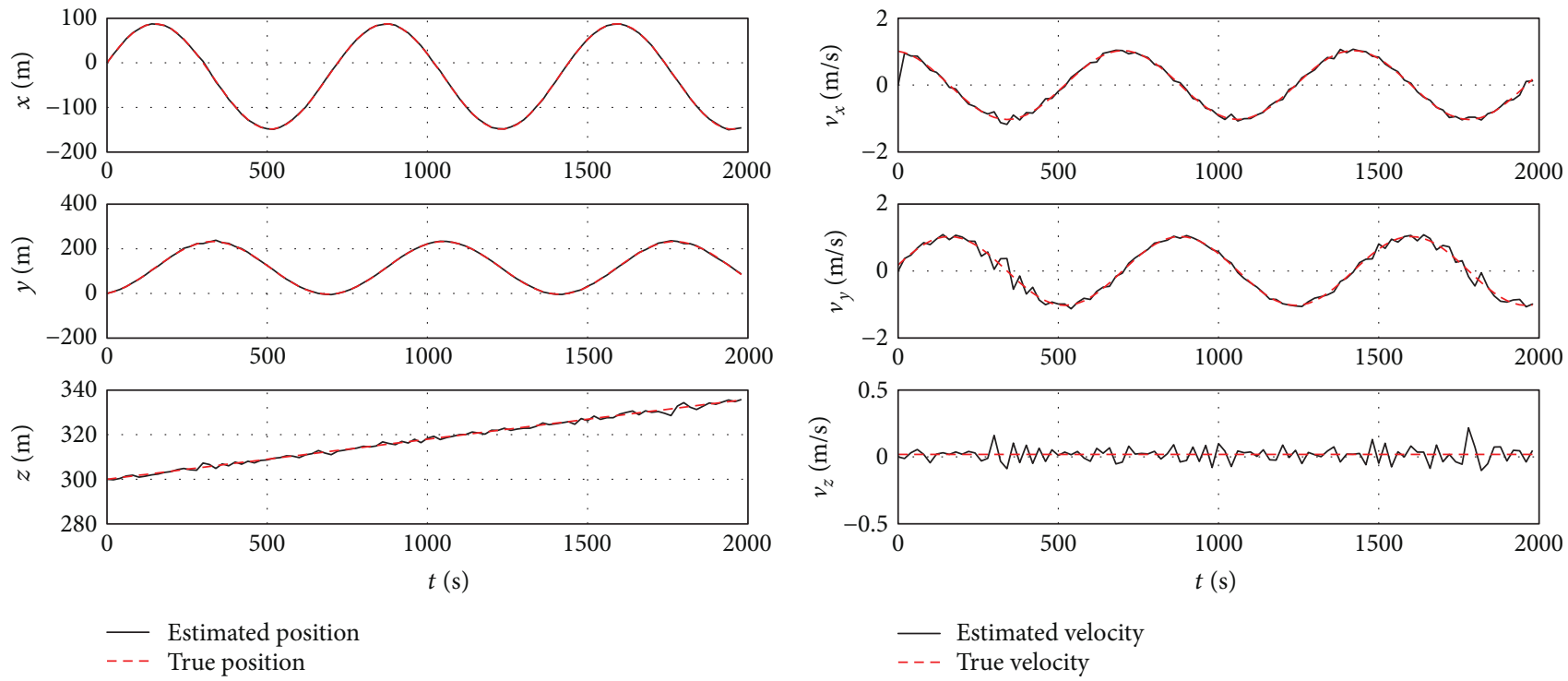

(a)

(b)

Figure 7: Comparison of the true and estimated values in general formation. (a) True and estimated position. (b) True and estimated velocity.
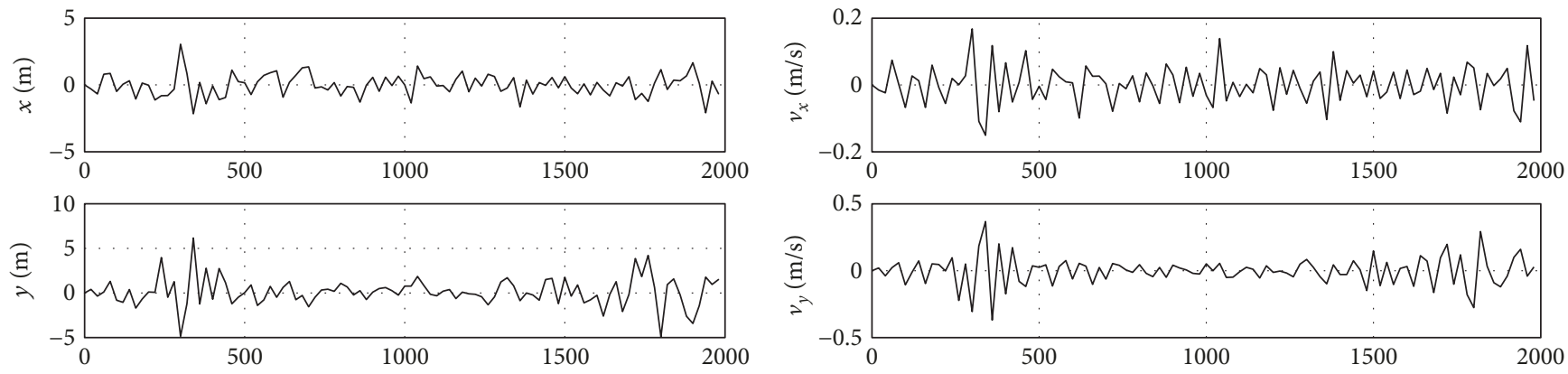

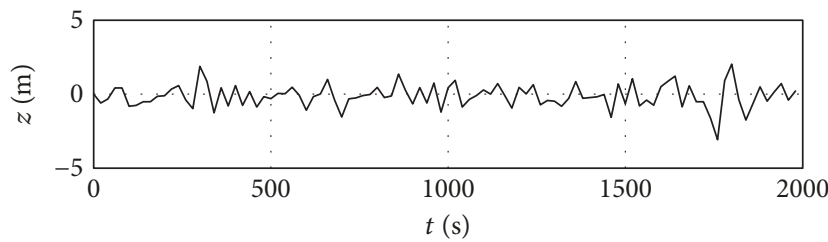

(a)

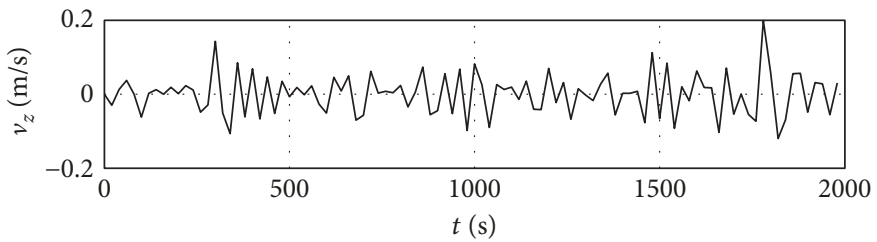

(b)

Figure 8: Estimation errors in general formation. (a) Position. (b) Velocity.

$$
\begin{aligned}
\mathbf{C}_{k} & :=\left[\begin{array}{cc}
\mathbf{C}_{1, k} & 0 \\
0 & \mathbf{I}_{4 \times 1}
\end{array}\right], \\
\mathbf{Y}_{1, k} & =\left[\gamma_{k}^{1,2}, \gamma_{k}^{2,3}, \gamma_{k}^{3,4}\right]^{\mathrm{T}}, \\
\mathbf{Y}_{2, k} & :=\left[\varphi_{1, k}, \varphi_{2, k}, \varphi_{3, k}, \varphi_{4, k}\right]^{\mathrm{T}} .
\end{aligned}
$$

Two examples are presented to verify the effectiveness of the proposed algorithm. In the first example, we identify the effect of the proposed algorithm in the optimal formation. In the second example, the validity of this algorithm is demonstrated in a general formation.
6.1. Example in Optimal Formation. In this example, the USVs are in the optimal formation. That is to say, four USVs are on the vertices of the square centered at the target. The parameters of the USVs and the target are shown in Table 2. The true and estimated trajectories of the target are shown in Figure 3. It can be seen that the target is well positioned. Figure 4 shows the comparison of the true and estimated values. The estimation errors of position and velocity are illustrated in Figure 5. These results demonstrate that the proposed algorithm can simultaneously estimate the position and velocity of the target in optimal formation.

6.2. Example in General Formation. In this example, the parameters of the target are the same as the parameters in 
TABLE 4: The max and average estimation errors.

\begin{tabular}{lcccc}
\hline \multirow{2}{*}{ Error } & \multicolumn{2}{c}{ Optimal formation } & \multicolumn{2}{c}{ General formation } \\
& Max & Average & Max & Average \\
\hline$x$ & 1.50 & 0.46 & 3.04 & 0.62 \\
$y$ & 1.73 & 0.47 & 6.14 & 1.10 \\
$z$ & 0.90 & 0.33 & 2.03 & 0.60 \\
$v_{x}$ & 0.097 & 0.033 & 0.168 & 0.045 \\
$v_{y}$ & 0.102 & 0.034 & 0.367 & 0.078 \\
$v_{z}$ & 0.082 & 0.024 & 0.199 & 0.044 \\
\hline
\end{tabular}

Table 2 and the USV s are in a general formation. The parameters of the USVs are shown in Table 3.

Figures 6-8 show the true and estimated trajectories of the target, comparison of the true and estimated values, and the estimation errors, respectively. The max and average values of absolute estimation errors in optimal and general formations are shown in Table 4. Simulation results show that the target is well positioned and the velocity of the target is effectively estimated.

\section{Conclusion}

In this paper, we consider the problem on how to simultaneously estimate the velocity and position of the target for multi-USV positioning system. Firstly, we formulate the MLBL system in a linear discrete-time system without direct feedthrough. In this system, the velocity and position of the target are seen as the input and state, respectively. Then, we propose a three-step minimum variance unbiased SISE algorithm to simultaneously estimate the velocity and position. The unbiased SISE conditions for this system are analysed. Finally, simulation results show the correctness of the problem formulation and the effectiveness of the algorithm. Besides, the existence condition for asymptotic stability and the experimental validation of the proposed algorithm may be explored and demonstrated in the future work.

\section{Data Availability}

The data used to support the findings of this study are available from the corresponding author upon request.

\section{Conflicts of Interest}

The authors declare that they have no conflicts of interest.

\section{Acknowledgments}

This work was supported by the National Natural Science Foundation of China under Grants 61733014 and 61703326.

\section{References}

[1] S. Sukkarieh, E. M. Nebot, and H. F. Durrant-Whyte, "A high integrity IMU/GPS navigation loop for autonomous land vehicle applications," IEEE Transactions on Robotics \& Automation, vol. 15, no. 3, pp. 572-578, 1999.
[2] X. Yun, E. R. Bachmann, R. B. McGhee et al., "Testing and evaluation of an integrated GPS/INS system for small AUV navigation," IEEE Journal of Oceanic Engineering, vol. 24, no. 3, pp. 396-404, 1999.

[3] M. Moradi, J. Rezazadeh, and A. S. Ismail, "A reverse localization scheme for underwater acoustic sensor networks," Sensors, vol. 12, no. 4, pp. 4352-4380, 2012.

[4] M. Erol-Kantarci, H. T. Mouftah, and S. Oktug, "A survey of architectures and localization techniques for underwater acoustic sensor networks," IEEE Communications Surveys \& Tutorials, vol. 13, no. 3, pp. 487-502, 2011.

[5] A. D. Waite, Sonar for Practising Engineers, John Wiley and Sons, London, UK, 3rd edition, 2002.

[6] H.-P. Tan, R. Diamant, W. K. G. Seah, and M. Waldmeyer, "A survey of techniques and challenges in underwater localization," Ocean Engineering, vol. 38, no. 14-15, pp. 16631676, 2011.

[7] E. Olson, J. J. Leonard, and S. Teller, "Robust range-only beacon localization," IEEE Journal of Oceanic Engineering, vol. 31, no. 4, pp. 949-958, 2006.

[8] J. C. Kinsey, R. M. Eustice, and L. L. Whitcomb, “A survey of underwater vehicle navigation: Recent advances and new challenges," in IFAC Conference of Manoeuvering and Control of Marine Craft, pp. 1-12, Lisbon, Portugal, September 2006.

[9] F. Arrichiello, H. K. Heidarsson, and G. S. Sukhatme, "Opportunistic localization of underwater robots using drifters and boats," in 2012 IEEE International Conference on Robotics and Automation, pp. 5307-5314, Saint Paul, MN, USA, May 2012.

[10] J. Vaganay, J. J. Leonard, J. A. Curcio, and J. S. Willcox, "Experimental validation of the moving long base-line navigation concept," in 2004 IEEE/OES Autonomous Underwater Vehicles (IEEE Cat. No.04CH37578), pp. 59-65, Sebasco, ME, USA, June 2004.

[11] J. Curcio, J. Leonard, J. Vaganay et al., "Experiments in moving baseline navigation using autonomous surface craft," in Proceedings of OCEANS 2005 MTS/IEEE, vol. 1, pp. 730-735, Washington, DC, USA, September 2005.

[12] A. Folk, B. Armstrong, E. Wolbrecht, H. F. Grip, M. Anderson, and D. Edwards, "Autonomous underwater vehicle navigation using moving baseline on a target ship," in OCEANS 2010 MTS/IEEE SEATTLE, pp. 1-7, Seattle, WA, USA, September 2010.

[13] S. Martinez and F. Bullo, "Optimal sensor placement and motion coordination for target tracking," Automatica, vol. 42, no. 4, pp. 661-668, 2006.

[14] Y. Oshman and P. Davidson, "Optimization of observer trajectories for bearings-only target localization," IEEE Transactions on Aerospace and Electronic Systems, vol. 35, no. 3, pp. 892902, 1999.

[15] K. B. Purvis, K. J. Astrom, and M. Khammash, "Estimation and optimal configurations for localization using cooperative UAVs," IEEE Transactions on Control Systems Technology, vol. 16, no. 5, pp. 947-958, 2008.

[16] W. Yan, W. Chen, R. Cui, and H. Li, "Optimal distance between mobile buoy and target for moving long baseline positioning system," Journal of Navigation, vol. 68, no. 04, pp. 809826, 2015.

[17] L. Wang, Z. Wang, Q. L. Han, and G. Wei, "Event-based variance-constrained $H \infty$ filtering for stochastic parameter systems over sensor networks with successive missing 
measurements," IEEE Transactions on Cybernetics, vol. 48, no. 3, pp. 1007-1017, 2018.

[18] L. Wang, Z. Wang, T. Huang, and G. Wei, “An event-triggered approach to state estimation for a class of complex networks with mixed time delays and nonlinearities," IEEE Transactions on Cybernetics, vol. 46, no. 11, pp. 2497-2508, 2016.

[19] S. Liu, G. Wei, Y. Song, and D. Ding, "Set-membership state estimation subject to uniform quantization effects and communication constraints," Journal of the Franklin Institute, vol. 354, no. 15, pp. 7012-7027, 2017.

[20] R. Cui, S. Sam Ge, B. Voon Ee How, and Y. Sang Choo, "Leader-follower formation control of underactuated autonomous underwater vehicles," Ocean Engineering, vol. 37, no. 17-18, pp. 1491-1502, 2010.

[21] R. Cui, Y. Li, and W. Yan, "Mutual information-based multiAUV path planning for scalar field sampling using multidimensional RRT," IEEE Transactions on Systems, Man, and Cybernetics: Systems, vol. 46, no. 7, pp. 993-1004, 2016.

[22] F. Gustafsson and F. Gunnarsson, "Mobile positioning using wireless networks: possibilities and fundamental limitations based on available wireless network measurements," IEEE Signal Processing Magazine, vol. 22, no. 4, pp. 41-53, 2005.

[23] A. Alcocer, P. Oliveira, and A. Pascoal, "Study and implementation of an EKF GIB-based underwater positioning system," Control Engineering Practice, vol. 15, no. 6, pp. 689-701, 2007.

[24] P. Batista, C. Silvestre, and P. Oliveira, "Sensor-based long baseline navigation: observability analysis and filter design," Asian Journal of Control, vol. 16, no. 4, pp. 974-994, 2014.

[25] D. Fox, W. Burgard, H. Kruppa, and S. Thrun, "A probabilistic approach to collaborative multi-robot localization," Autonomous Robots, vol. 8, no. 3, pp. 325-344, 2000.

[26] A. Howard, M. J. Matark, and G. S. Sukhatme, "Localization for mobile robot teams using maximum likelihood estimation," in IEEE/RSJ International Conference on Intelligent Robots and System, vol. 1, pp. 434-439, Lausanne, Switzerland, September 2002.

[27] G. C. Calafiore, L. Carlone, and M. Wei, "A distributed technique for localization of agent formations from relative range measurements," IEEE Transactions on Systems, Man, and Cybernetics - Part A: Systems and Humans, vol. 42, no. 5, pp. 1065-1076, 2012.

[28] Y.-T. Chan, H. Y. C. Hang, and P.-c. Ching, "Exact and approximate maximum likelihood localization algorithms," IEEE Transactions on Vehicular Technology, vol. 55, no. 1, pp. 10-16, 2006.

[29] T. Li, J. Su, W. Liu, and J. M. Corchado, “Approximate Gaussian conjugacy: parametric recursive filtering under nonlinearity, multimodality, uncertainty, and constraint, and beyond," Frontiers of Information Technology \& Electronic Engineering, vol. 18, no. 12, pp. 1913-1939, 2017.

[30] P. K. Kitanidis, "Unbiased minimum-variance linear state estimation," Automatica, vol. 23, no. 6, pp. 775-778, 1987.

[31] S. Gillijns and B. De Moor, "Unbiased minimum-variance input and state estimation for linear discrete-time systems," Automatica, vol. 43, no. 1, pp. 111-116, 2007.

[32] S. Gillijns and B. De Moor, "Unbiased minimum-variance input and state estimation for linear discrete-time systems with direct feedthrough," Automatica, vol. 43, no. 5, pp. 934937, 2007.
[33] T. Floquet and J.-P. Barbot, "State and unknown input estimation for linear discrete-time systems," Automatica, vol. 42, no. 11, pp. 1883-1889, 2006.

[34] S. Z. Yong, M. Zhu, and E. Frazzoli, "A unified filter for simultaneous input and state estimation of linear discrete-time stochastic systems," Automatica, vol. 63, pp. 321-329, 2016.

[35] J. Su, B. Li, and W. H. Chen, "On existence, optimality and asymptotic stability of the Kalman filter with partially observed inputs," Automatica, vol. 53, pp. 149-154, 2015.

[36] H. Fang, Y. Shi, and J. Yi, "On stable simultaneous input and state estimation for discrete-time linear systems," International Journal of Adaptive Control and Signal Processing, vol. 25, no. 8, pp. 671-686, 2011.

[37] R. V. Hogg, J. McKean, and A. T. Craig, Introduction to Mathematical Statistics, Pearson Prentice Hall, Upper Saddle River, NJ, USA, 6th edition, 2005.

[38] T. I. Fossen, Marine Control Systems: Guidance, Navigation and Control of Ships, Rigs and Underwater Vehicles, Marine Cybernetics, Trondheim, Norway, 2002.

[39] D. Moreno-Salinas, A. Pascoal, and J. Aranda, "Optimal sensor placement for underwater positioning with uncertainty in the target location," in 2011 IEEE International Conference on Robotics and Automation, pp. 2308-2314, Shanghai, China, 2011.

[40] D. B. Jourdan and N. Roy, "Optimal sensor placement for agent localization," ACM Transactions on Sensor Networks, vol. 4, no. 3, pp. 1-40, 2008.

[41] M. S. Bazaraa, H. D. Sherali, and C. M. Shetty, Nonlinear Programming: Theory and Algorithms, John Wiley and Sons, Hoboken, NJ, USA, 3rd edition, 2013. 


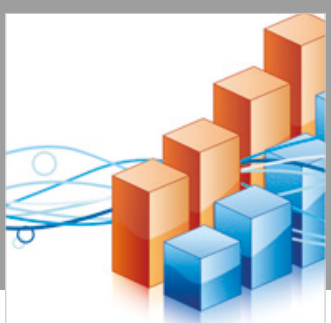

Advances in

Operations Research

\section{-n-m}
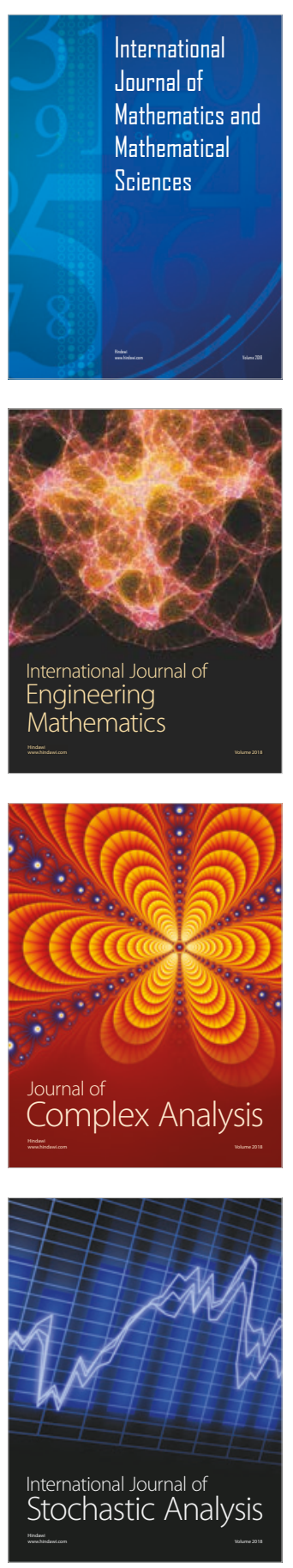
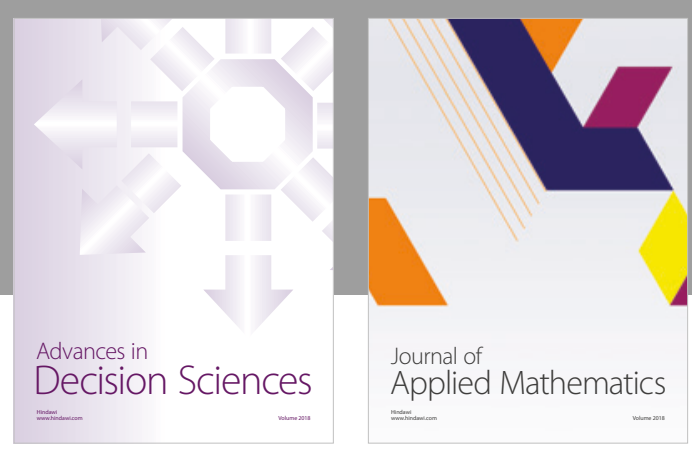

Journal of

Applied Mathematics
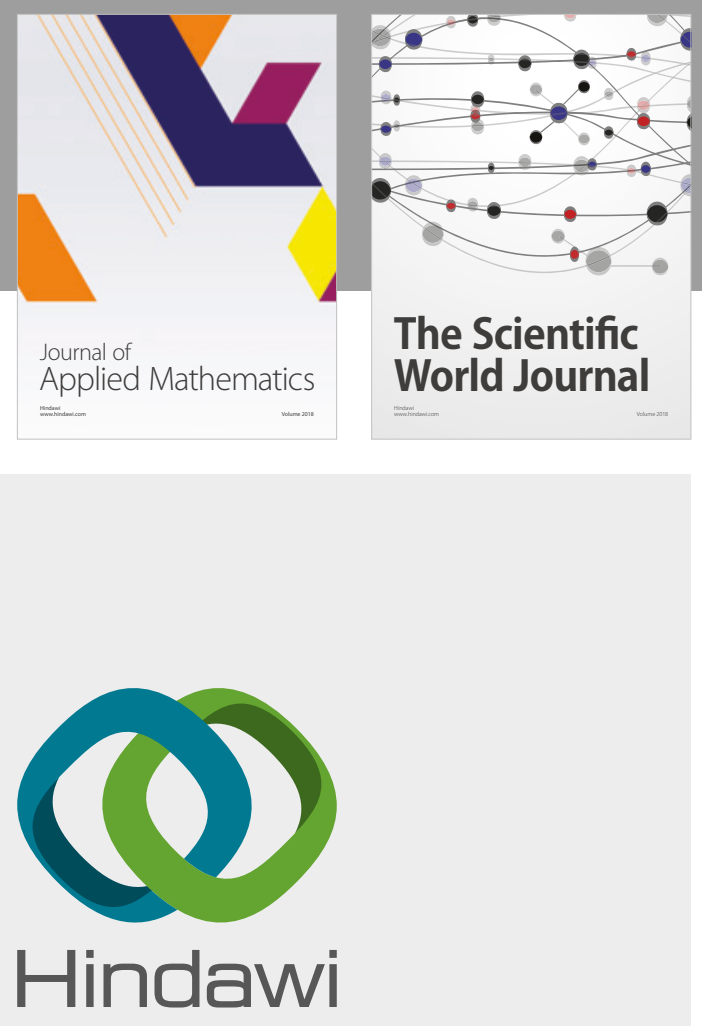

Submit your manuscripts at

www.hindawi.com

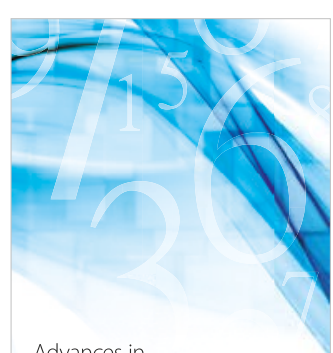

Advances in
Numerical Analysis
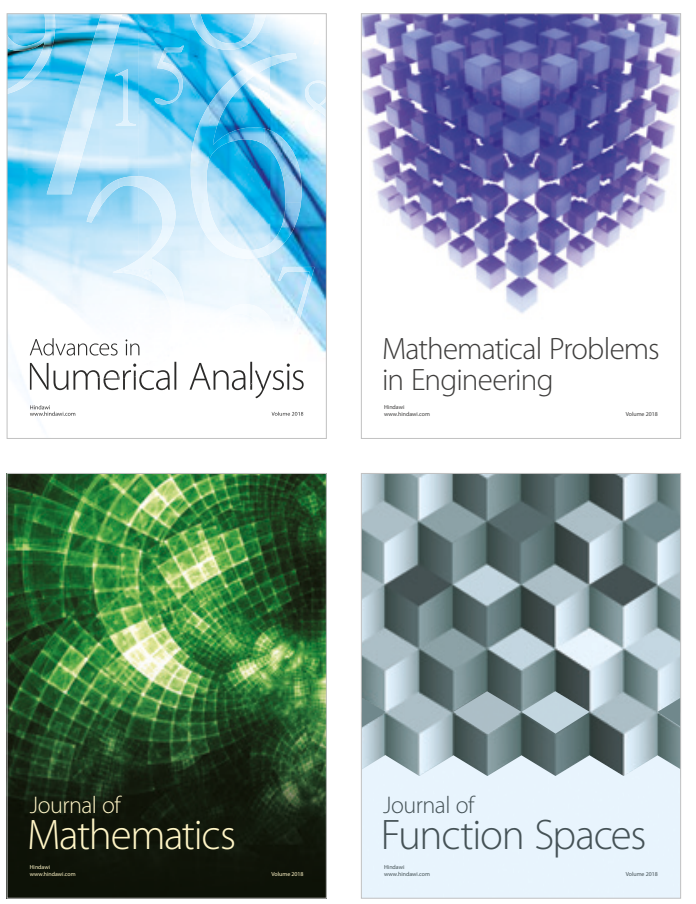

Mathematical Problems in Engineering

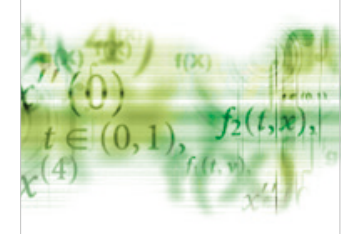

International Journal of

Differential Equations

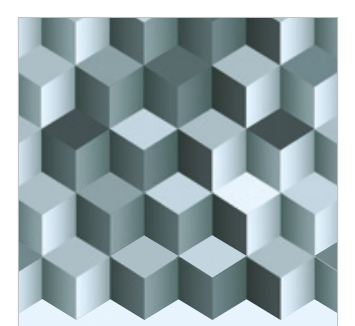

Journal of

Function Spaces
The Scientific

World Journal

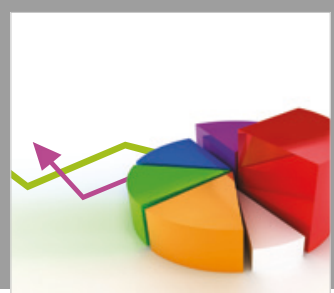

Journal of

Probability and Statistics
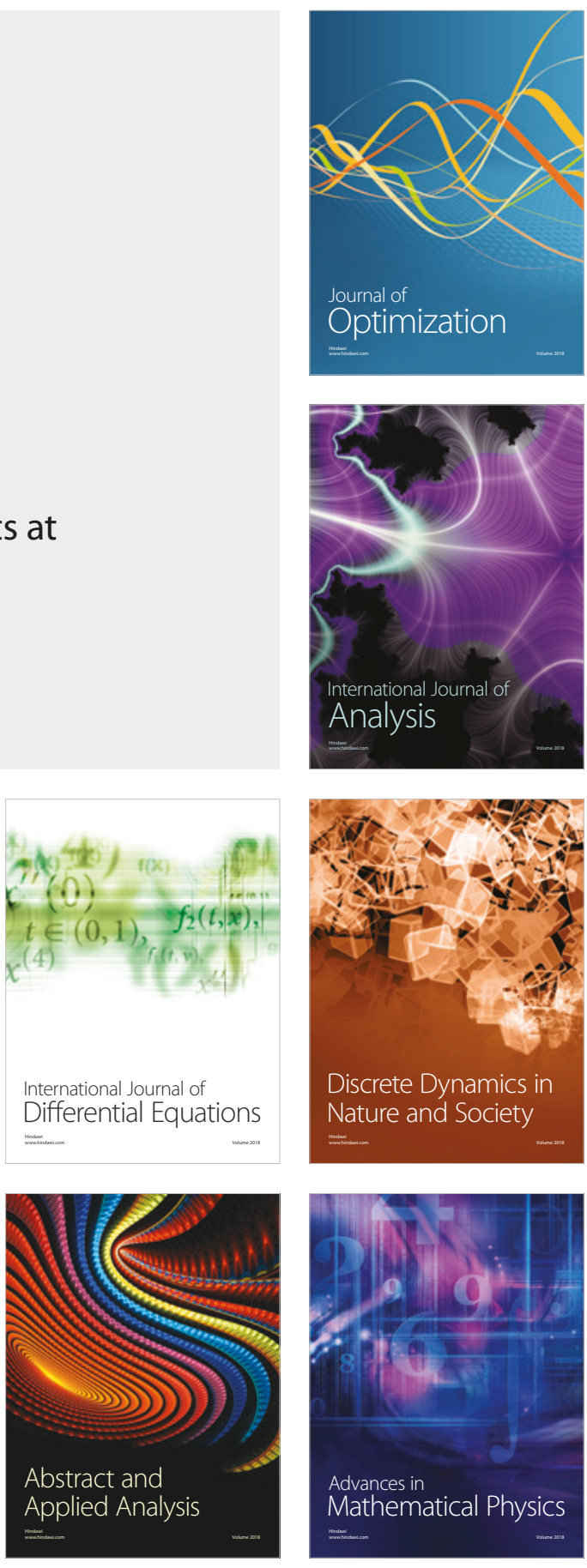